\title{
Analysis and modelling of recurrent solar flares observed with Hinode/EIS on March 9, 2012^
}

\author{
V. Polito ${ }^{1}$, G. Del Zanna ${ }^{1}$, G. Valori ${ }^{2}$, E. Pariat ${ }^{3}$, H. E. Mason ${ }^{1}$, J. Dudík ${ }^{4}$, and M. Janvier ${ }^{5}$ \\ ${ }^{1}$ Department of Applied Mathematics and Theoretical Physics, CMS, University of Cambridge, Wilberforce Road, \\ Cambridge CB3 0WA, UK \\ e-mail: vp323@cam.ac.uk \\ 2 UCL Mullard Space Science Laboratory, Holmbury St. Mary, Dorking, Surrey RH5 6NT, UK \\ 3 LESIA, Observatoire de Paris, PSL Research University, CNRS, Sorbonne Universités, UPMC Univ. Paris 06, Univ. Paris Diderot, \\ Sorbonne Paris Cité, 5 place Jules Janssen, 92195 Meudon, France \\ 4 Astronomical Institute, Academy of Sciences of the Czech Republic, 25165 Ondřejov, Czech Republic \\ 5 Institut d'Astrophysique Spatiale, CNRS, Univ. Paris-Sud, Université Paris-Saclay, Bât. 121, 91405 Orsay Cedex, France
}

Received 12 September 2016 / Accepted 22 December 2016

\begin{abstract}
Three homologous C-class flares and one last M-class flare were observed by both the Solar Dynamics Observatory (SDO) and the Hinode EUV Imaging Spectrometer (EIS) in the AR 11429 on March 9, 2012. All the recurrent flares occurred within a short interval of time (less than $4 \mathrm{~h}$ ), showed very similar plasma morphology and were all confined, until the last one when a large-scale eruption occurred. The C-class flares are characterized by the appearance, at approximatively the same locations, of two bright and compact footpoint sources of $\approx 3-10 \mathrm{MK}$ evaporating plasma, and a semi-circular ribbon. During all the flares, the continuous brightening of a spine-like hot plasma $(\approx 10 \mathrm{MK})$ structure is also observed. Spectroscopic observations with Hinode/EIS are used to measure and compare the blueshift velocities in the Fe XXIII emission line and the electron number density at the flare footpoints for each flare. Similar velocities, of the order of $150-200 \mathrm{~km} \mathrm{~s}^{-1}$, are observed during the C2.0 and C4.7 confined flares, in agreement with the values reported by other authors in the study of the last M1.8 class flare. On the other hand, lower electron number densities and temperatures tend to be observed in flares with lower peak soft X-ray flux. In order to investigate the homologous nature of the flares, we performed a non-linear force-free field (NLFFF) extrapolation of the 3D magnetic field configuration in the corona. The NLFFF extrapolation and the Quasi-Separatrix Layers (QSLs) provide the magnetic field context which explains the location of the kernels, spine-like hot plasma and semi-circular brightenings observed in the (non-eruptive) flares. Given the absence of a coronal null point, we argue that the homologous flares were all generated by the continuous recurrence of bald patch reconnection.
\end{abstract}

Key words. Sun: flares - Sun: UV radiation - techniques: spectroscopic - magnetic fields - methods: numerical

\section{Introduction}

Although major observational advances and significant progress in theoretical modelling have been achieved in the last few decades, we still lack a definitive model for solar flares. The standard model of flares in 2D (CSHKP; Carmichael 1964; Sturrock 1968; Hirayama 1974; Kopp \& Pneuman 1976) proposes that flares are driven by magnetic reconnection in the corona. The energy release due to reconnection results in heating of the local plasma, bulk kinetic energy and wave generation, although it is still unclear how the energy is partitioned between different processes. In all cases, the energy is transported towards the chromosphere at the flare footpoints, also known as kernels, where the plasma is heated to very high temperatures (above $10 \mathrm{MK}$ ), and evaporates along the field lines due to the overpressure (i.e. chromospheric evaporation). The 2D model succeeds in explaining the observed chromospheric brightenings and high temperature upflows at the flare footpoints, as well as particle acceleration and the thermal cooling of loops. However, it fails to reproduce some more detailed features that can only be explained by 3D models of eruptive flares, such as the strong-to-weak evolution of the

\footnotetext{
$\star$ The movie associated to Fig. 2 is available at http://www . aanda.org
}

shear of flare loops (Aulanier et al. 2012), the apparent slipping motion of the flare footpoints (Janvier et al. 2013; Dudík et al. 2014,2016 ) and the $j$-shaped structure of the ribbons (see e.g. Janvier et al. 2015, and references therein). Also, in the 3D models, magnetic reconnection can happen even in the absence of a null point: rather it is associated with finite-volume regions where the magnetic connectivity is characterized by strong gradients, called quasi-separatrix layers (QSLs; Priest \& Démoulin 1995; Demoulin et al. 1996; Titov et al. 2002). While there is a general consensus that magnetic reconnection is the energy release mechanism for flares, the details of the energy conversion and transport through the corona are still strongly debated. Comparing the theoretical models with observations is complicated by the fact that we cannot observe the energy release directly. A possible approach to this problem is to observe the result of the heating, that is, plasma observables in extreme UV (EUV) and X-ray wavelengths such as flows, density, temperature, emission measure and electron distribution. Several authors have compared observations with modelling in order to infer evidence supporting a particular flare model, between thermal conduction, thick-target or Afvenic wave models (see e.g. Petkaki et al. 2012; Doschek et al. 2015; Battaglia et al. 2015; Polito et al. 2016). One of the key observables is the blueshift of spectral lines revealing upflows at the loop footpoints during 
the chromospheric evaporation phase. This was first observed in the soft X-ray lines with SOLFLEX (Doschek et al. 1979) and the solar maximum mission (SMM; Antonucci et al. 1982). These lines (8-25 MK) showed strong blue-asymmetric profiles, in contrast to the theoretical predictions of completely blueshifted line profiles (Emslie \& Alexander 1987). The chromospheric evaporation phase in flares has subsequently been extensively observed with the Coronal Diagnostic Spectrometer (CDS; Harrison et al. 1995) on board SoHO and the EUV Imaging Spectrometer (EIS; Culhane et al. 2007) on board Hinode. Similar to the earlier studies, many CDS and EIS observations (e.g. Teriaca et al. 2003; Brosius 2003; Milligan et al. 2006) still showed strong asymmetries in the high temperature line profiles (Fe XIX with CDS, Fe XXIII and Fe XXIV with EIS). These asymmetries were often interpreted as due to a superposition of plasma upflows at different velocities along the line of sight (Warren \& Doschek 2005; Reeves et al. 2007). Totally shifted symmetrical profiles were however sometimes observed (Del Zanna et al. 2006, 2011a; Brosius 2013), in agreement with theory. Our understanding of chromospheric evaporation has been considerably improved since the launch of the IRIS satellite (De Pontieu et al. 2014) in 2013. In particular, simultaneous joint observations with both EIS (in Fe XXIII) and IRIS (in Fe XXI) have confirmed that the asymmetric profiles seen with EIS are mostly due to the limited spatial resolution of EIS, since IRIS observed totally shifted Fe XXI profiles during the entire observation (Polito et al. 2016). On the other hand, compared to IRIS, EIS has the advantage of observing many spectral lines formed at coronal and flare temperatures, which can provide useful plasma diagnostics during flares (see e.g. the observational review by Milligan 2015). For instance, in the observation of a small B-class flare by Del Zanna et al. (2011a), the authors reported a comprehensive study of chromospheric evaporation, cooling and evolution of the plasma based on the analysis of several spectral lines with EIS.

One of the unsolved questions in solar flare models is understanding whether the same mechanism is responsible for large and small size events. Kahler (1982) suggested that there is a statistical correlation between solar flare energy release and the magnitude of any measured flare energy manifestation. Several statistical studies have been dedicated to comparing the plasma parameters of a large number of flares over time (e.g. Feldman et al. 1996; Battaglia et al. 2005; Hannah et al. 2011). For instance, Feldman et al. (1996) studied the correlation between soft X-ray flare class and emission measure with electron temperature for 868 flares of X-ray class A2 to X2. They found that the logaritmic of the flux measure in GOES $1-8 \AA$ or 0.5-4 $\AA$ channels and the EM measured by either detector is linearly proportional to the electron temperature.

It should be noted that flare events happening in very different plasma environments are difficult to compare directly. In fact, each flare can show different plasma parameters because of the different initial conditions in the active region where it occurred. The so-called recurrent or homologous flares are particularly interesting events, where a similar magnetic field configuration is reformed over time. Several authors have focused on analysing the magnetic field structure and evolution in recurrent flares. Some of the studies suggest that the flares are caused by the continuous emergence of new magnetic flux in the active region (e.g. Nitta \& Hudson 2001). Other authors concluded that persistent shearing motions could trigger the energy release in homologous flares (e.g. Romano et al. 2015). Although there are several imaging and magnetic field observations of recurrent flares, there have not been many spectroscopic observations to date. One of the main issues is associated with the difficulty of observing the exact location of the flare footpoints for a long period of time within the limited field of view of spectroscopic instruments.

A sequence of four recurrent flares in the active region (AR) NOAA 11429 was observed by EIS on March 9, 2012. The EIS instrument was in a raster mode and could observe both flare loop footpoints. Spectroscopic observations of recurrent flares in the same active region (and covering both footpoint locations) offer the unique possibility of comparing the physical observables in flares of different size taking place in a similar plasma environment. In particular, we aim to address the following questions:

- How do the plasma parameters (flows, density, temperature) vary in flares of increasing energy?

- Is there a difference in the upflows observed at the two footpoints during the chromospheric evaporation phase?

- How does the timing of the EIS observations (slit position during the raster) affect the measurement of the upflows during the chromospheric evaporation process?

- What causes the homologous flares and how does the magnetic field structure evolve?

The first three questions can be addressed by combining multiwavelength imaging from the SDO/Atmospheric Imaging Assembly (AIA; Boerner et al. 2012) with spectroscopic observations from EIS. In order to study the magnetic field structure during the recurrent flares, we analysed data from the SDO/Helioseismic and Magnetic Imager (HMI; Scherrer et al. 2012) and performed a non linear force-free field (NLFFF) extrapolation in the active region under study.

The paper is structured as follows: Sect. 2 introduces the context of the AIA and EIS observations and the flare events. A detailed analysis of the blueshifts observed by EIS is then presented in Sect. 3. Sections 4 and 5 provide density and temperature diagnostics based on the use of spectroscopic and imaging data. An analysis of the magnetic topology of the active region during the recurrent flares and the results of the extrapolation are then presented in Sect. 6. Finally, we discuss and summarize our results in Sect. 7 .

\section{Spectroscopic and imaging observations of the recurrent flares}

Active region NOAA 11429 was a highly complex $\beta \gamma \delta$ region that produced many energetic events during the interval March 7-11, 2012. The magnetic evolution of this region was analysed by several authors (e.g. Chintzoglou et al. 2015; Syntelis et al. 2016; Kouloumvakos et al. 2016; Patsourakos et al. 2016).

A sequence of five recurrent flares occurred in the AR 11429 on March 9, 2012 from around 00:00 UT to 04:18 UT, as shown in the GOES light curves in Fig. 1. The first four C-class flares (C1.0, C2.0, C4.7 and C1.2) were all confined. The last M-class eruptive flare started at 03:22 UT, reached a first maximum in the soft X-ray flux at around 03:27 UT (M1.8 class) and, after two small dips in intensity, increased up to about M6.3 (see Fig. 1). The EIS spectrometer observed three of the C-class flares and the first part of the M-class flare, as indicated by the red arrows in Fig. 1.

The M-class flare is a well-studied event. A detailed analysis of the EIS observations of the M1.8 flare was presented 


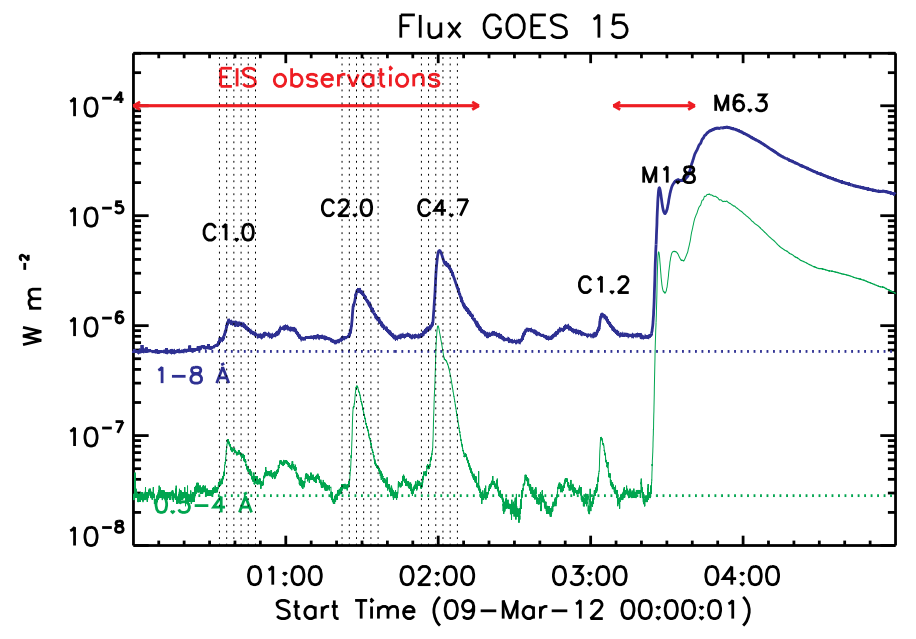

Fig. 1. Soft X-ray light curves of the recurrent flares on March 9, 2012 observed by GOES in the $0.5-4 \AA$ and $1-8 \AA$ channels. The vertical dotted lines indicate the time of the EIS rasters that we analysed in this work, as explained in Sect. 2.2. The red arrows indicate the period of time when EIS was observing the flaring AR. See text for more details.

Table 1. Time of the recurrent flares as observed by GOES.

\begin{tabular}{ccccc}
\hline \hline Flare & $\begin{array}{c}\text { Start } \\
(\mathrm{UT})\end{array}$ & $\begin{array}{c}\text { Peak } \\
(\mathrm{UT})\end{array}$ & $\begin{array}{c}\text { End } \\
(\mathrm{UT})\end{array}$ & Observed by EIS \\
\hline $\mathrm{C} 1.0$ & $00: 34$ & $00: 37$ & $00: 46$ & $\checkmark$ \\
$\mathrm{C} 2.0$ & $01: 23$ & $01: 28$ & $01: 34$ & $\checkmark$ \\
$\mathrm{C} 4.7$ & $01: 55$ & $02: 00$ & $02: 06$ & $\checkmark$ \\
$\mathrm{C} 1.2$ & $03: 01$ & $03: 04$ & $03: 08$ & $\boldsymbol{X}$ \\
$\mathrm{M} 1.8 / 6.3$ & $03: 22$ & $03: 27 / 03: 53$ & $04: 18$ & $\checkmark$ \\
\hline
\end{tabular}

by Doschek et al. (2013). This eruptive flare was also studied by Simões et al. (2013), who analysed the strong contracting motions of peripheral coronal loops during the flare impulsive phase. In addition, Hao et al. (2012) focused on studying the white-light emission produced during the flare.

In this work we mainly focus on the series of three C-class confined flares observed before the M-class flare by EIS. The vertical dotted lines in Fig. 1 indicate the times of the EIS rasters which were analysed in this work. The start, peak, and end times of all the flares are summarized in Table 1.

Section 2.1 provides an overview of the homologous flares (including the last eruptive one), as observed in the SDO/AIA multiwavelength images. Section 2.2 presents the details of the EIS spectroscopic observation of the three C-class confined flares.

\subsection{AlA and HMI observation}

The SDO/AIA and SDO/HMI magnetograms data were downloaded through the solarsoft VSO package and converted to level 1.5 images using the aia_prep and hmi_prep routines, respectively. The images were also corrected for solar rotation and aligned with the EIS images, as described in Sect. 2.2.

Figure 2 shows an overview of the AIA observation of the homologous flares in the 304 (left), 171 (middle) and 131 (right) $\AA$ filters. During flares, these filters are dominated by emission from plasma at $\approx 0.05 \mathrm{MK}, 1 \mathrm{MK}$ and $11 \mathrm{MK}$ respectively (e.g. O'Dwyer et al. 2010; Del Zanna et al. 2011b). The online Movie 1 shows the evolution of the recurrent flares over time, as observed by the $171 \AA$ and $131 \AA$ filters respectively.
The first row of Fig. 2 shows the first C1.0 class flare just after the peak, at around 00:38 UT. The flare ribbons can best be seen in the low temperature $304 \AA$ image, while the $131 \AA$ image shows the high temperature flare loops $(\approx 10 \mathrm{MK})$. The $304 \AA$ image also shows an elongated and dark filament structure following the polarity inversion line (PIL) along the whole AR. The filament appears to be composed of several segments that are constantly present until the major eruption occurs during the M-class flare. While the western arm has a single U-structure, the eastern arm extends northward as a collection of smaller fragments. The observations, including those at other wavelengths, do not allow us to discern if the fragmentation corresponds to an equally fragmented magnetic structure or rather is the effect of irregular absorption along the filament.

The second row in Fig. 2 shows the second C2.0 class flare, while the peak of the C4.7 class flare (at around 02:00 UT) is shown in the third row, with the field of view of the EIS spectrometer overlaid (indicated by the coloured yellow and white boxes). To better understand the context of the observed event, the intensity contours of the $304 \AA$ AIA image around 02:00 UT are overlaid on the line of sight magnetic field $\left(B_{\mathrm{LOS}}\right)$ map observed with SDO/HMI in Fig. 3. The overlay shows the complex morphology of the flare ribbons, with the negative polarity ribbon having a semi-circular shape. We can also observe two bright emission sources in the AIA $304 \AA$ intensity contours, which are located on opposite magnetic polarities and indicated as K1 and K2 in Figs. 2 and 3. We indicate these footpoint sources as kernels, since they represent the location where the chromospheric evaporation takes place (in the context of the 2D standard flare model) as better described in Sects. 2.2 and 3. This interpretation is corroborated by the simultaneity of the appearance of the two kernels in all three C-class flares (Fig. 2 and online movie), which indicates that the two locations are magnetically connected by loops created during the flare events.

Figure 2 also shows the presence of a spine-like feature, which is indicated by the white arrow in the $131 \AA$ image at around 02:00 UT (third row). This feature is observed to brighten up during all the recurrent flares. A more detailed analysis of the magnetic field structure associated with these features is presented in Sect. 6.

The AIA images in the fourth row of Fig. 2 show the eruptive M-class flare just after its peak. In these images we note that the flare loops and the bright spine-like structure form at approximately the same location as the confined flares.

\subsection{EIS observation}

On March 9, 2012, EIS was running a core_flare_tr120x120 study from around 00:02:39 UT to 02:16:04 UT on the AR 11429 , observing the sequence of the three C-class homologous flares over a field of view of $120^{\prime \prime} \times 120^{\prime \prime}$. From around 03:09:33 UT, the spectrometer then run an Atlas_30 fullspectrum study and caught the last eruptive M1.8 class flare. The timing of core_flare_tr120x120 and Atlas_30 observing studies is indicated by the first and second (from left to right) red arrows, respectively, in Fig. 1. The Atlas_30 study was analysed in detail by Doschek et al. (2013). In the following, we focus on the spectroscopic analysis of the first three confined flares but also compare our results with the diagnostics reported by Doschek et al. (2013). The EIS core_flare_tr120x120 study is a large raster including $30 \times 2^{\prime \prime}$ slit positions with a jump of $1^{\prime \prime}$ between each position. The exposure time is around $4 \mathrm{~s}$, resulting in a total raster cadence of around $3 \mathrm{~min}$. The study includes 

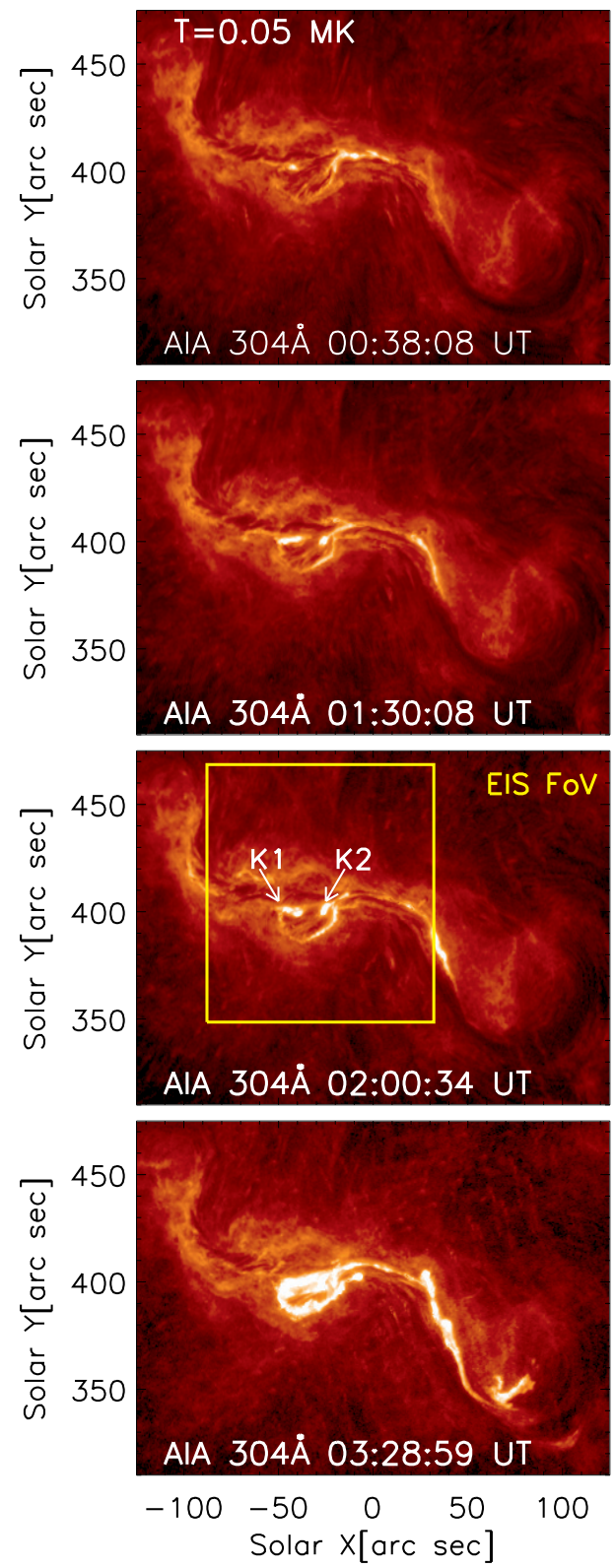
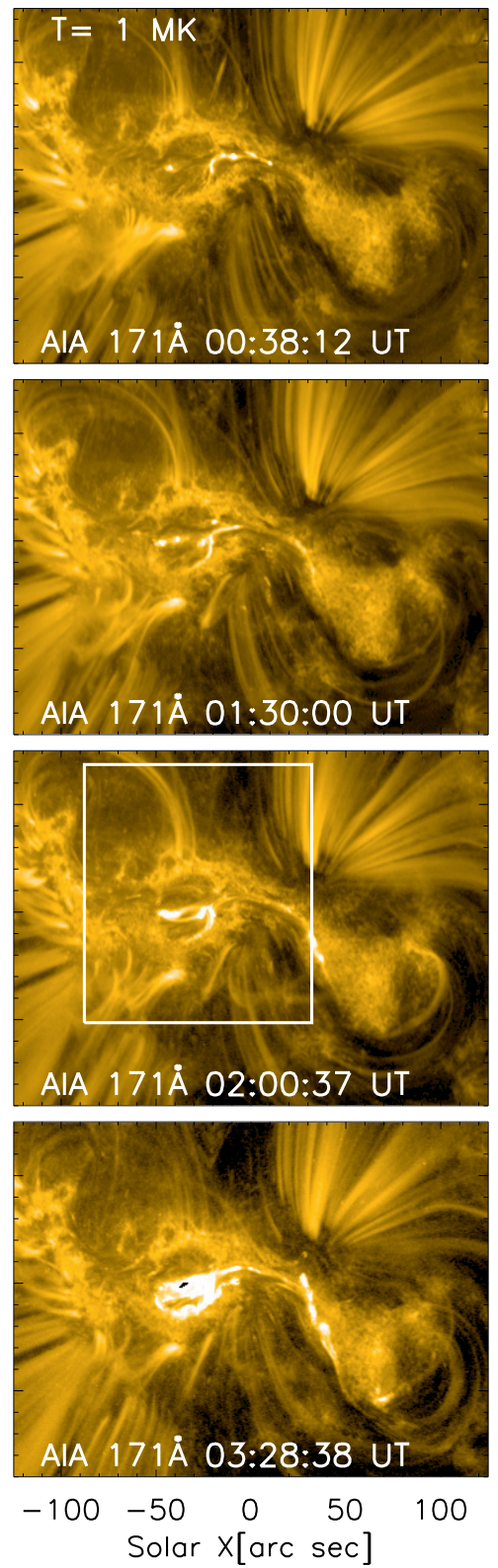
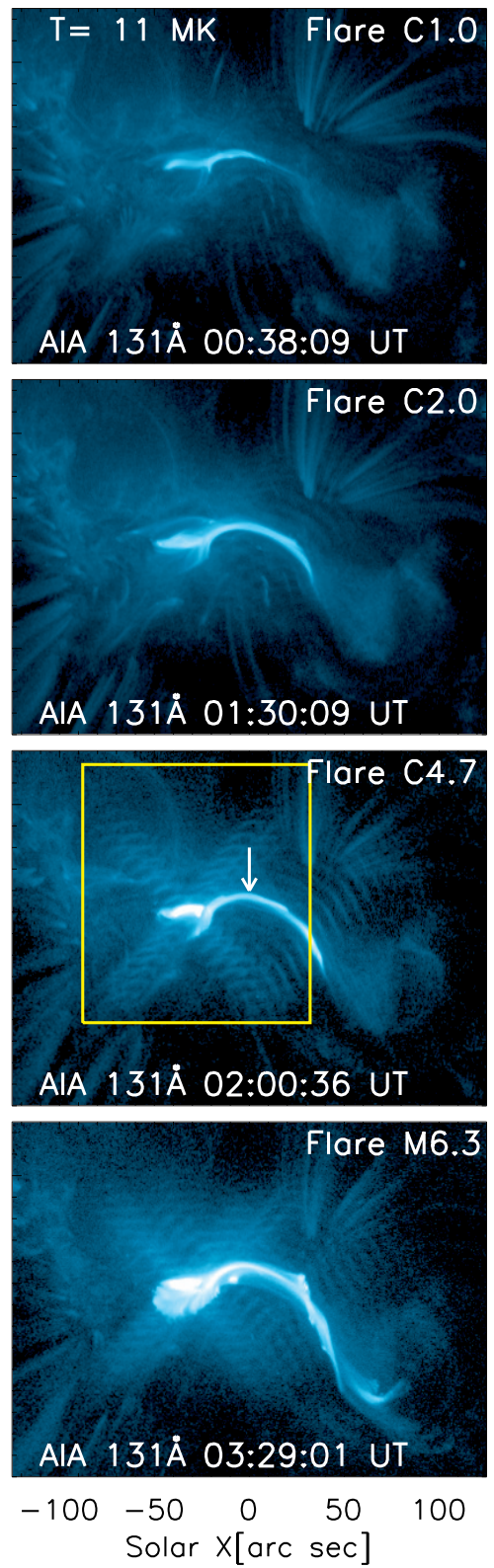

Fig. 2. Overview of the C-class recurrent flares on March 9, 2012 as observed by AIA in the following channels: 304 (left column), 171 (middle) and $131 \AA$ (right). The field of view of the EIS spectrometer is indicated by the yellow (and white in the middle column) boxes in the figure. In addition, the locations of the footpoints K1 and K2 (see Sect. 3) are indicated on the 304 A image in the third row. Finally, the small white arrows in the $131 \AA$ image in the third, fifth, and sixth rows indicate: the spine-like feature, the erupting flux rope, and the slipping motion of the southern flare ribbon respectively, as discussed in the text. The online movie shows the evolution of the 171 and $131 \AA$ AIA images over time. See text for discussion of the rows.

several spectral windows, but in this work we only analyse the spectral lines listed in Table 2. The high temperature Fe XXIV lines within the EIS spectral range are unfortunately not included in this observation.

In order to understand the context of the spectroscopic observations, we first align the EIS monochromatic intensity images with the AIA multiwavelength images. This can be carried out by comparing the AIA and EIS images, which are formed at similar temperatures and which therefore show plasma with the same morphology. In particular, we compared AIA 304, 211 , 335, and $131 \AA$ images with EIS raster images formed in the

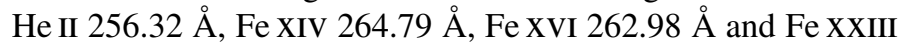
$263.78 \AA$ lines, respectively. The co-alignment between EIS and AIA at around 02:00 UT is shown in Fig. 4. The field of view of the AIA images in Fig. 4 is overlaid on Fig. 3 as context. The EIS intensity images were obtained by performing a Gaussian fit at each pixel in the raster using the $c$ fit_block solarsoft routine.

The flare ribbons are best seen in the cool $304 \AA$ (He II) emission in Fig. 4, while the 211 and $335 \AA$ images $(\approx 2$ and $3 \mathrm{MK})$ show that the two compact sources $\mathrm{K} 1$ and $\mathrm{K} 2$ and the spinelike feature noted in Sect. 2.1 are also visible in the EIS intensity contours. Hot flare loops visible in the Fe XXIII emission are formed between these sources, as shown in the $131 \AA$ panel of Fig. 4. This can also be best seen in Fig. 5, showing a zoom of the EIS Fe XXIII image of the hot C4.7 flare loop (LT indicates the loop-top) with the Fe XVI intensity contours of the K1 and $\mathrm{K} 2$ footpoints overlaid. Section 3 describes in detail the evolution of the high temperature $(\approx 3-10 \mathrm{MK})$ blueshifts at the same 


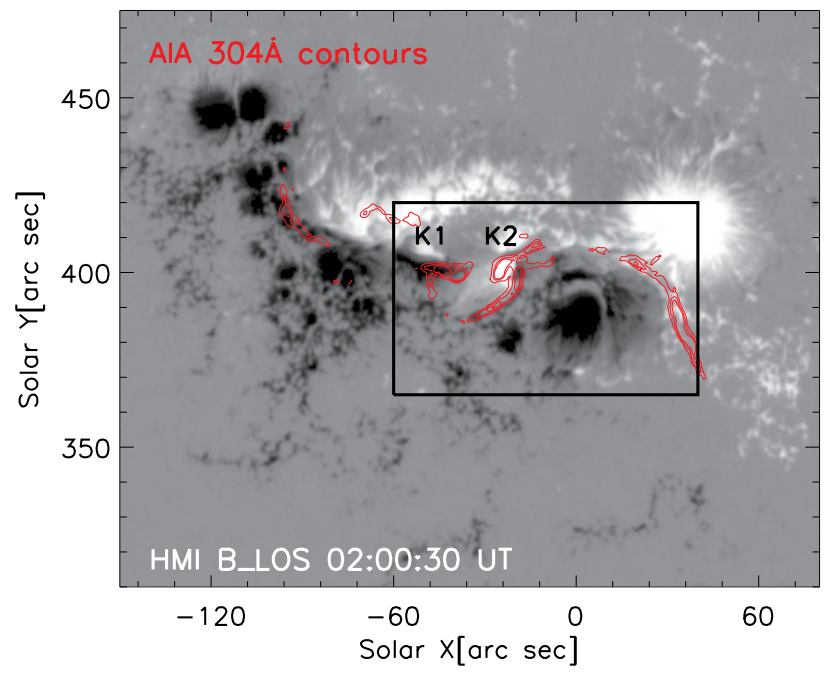

Fig. 3. SDO/HMI $B_{\text {LOS }}$ image of the AR 11429 during the peak of the C4.7 class flare. The intensity contours of the AIA $304 \AA$ image of the flare are overplotted in red. The position of the flare footpoints K1 and K2 (see Sect. 3) are also indicated, showing that the two footpoints are located in different magnetic polarities. The field of view of the AIA images in Fig. 4 is overlaid as a black boxed area.

compact footpoint locations $\mathrm{K} 1$ and $\mathrm{K} 2$ for the sequence of the three recurrent C-class flares observed by EIS. We only focus on the C4.7 and C2.0 class flares, as only weak Fe XXIII emission and evaporation are observed for the smallest $\mathrm{C} 1.0$ class flare.

\section{Evolution of the blueshifts}

In this section, we start discussing the observation of the $\mathrm{C} 4.7$ class flare, where the Fe XXIII line during the evaporation phase is strongest. The maximum upflows observed in the confined C4.7 and C2.0 class flares are summarized in Table 3, which also includes a comparison with the results of the analysis carried out by Doschek et al. (2013) for the M1 class eruptive flare. For this last flare, it is not possible to identify two clear footpoint sources as multiple complex footpoint regions are observed by the authors.

\subsection{Accuracy of blueshift measurements}

The measurements of the blueshifts require an accurate wavelength calibration that is complicated by the lack of photospheric reference lines in the EIS spectra. This is particularly difficult for the Fe XXIII $263.765 \AA$ high temperature line, which is not visible outside the flare region. In order to obtain a reference wavelength for the Fe XXIII spectra, we measured the centroid of the neighbouring Fe XVI $263.984 \AA$ line in a background quiet-Sun region, using a similar method to Polito et al. (2016). The calibration procedure was then repeated for each EIS raster analysed in this study to account for the periodic shift of the wavelength scale during the satellite orbital motion (Kamio et al. 2010). The error associated with the wavelength calibration is estimated to be within $\approx 5 \mathrm{~km} \mathrm{~s}^{-1}$ (e.g. Young et al. 2012). The errors associated with the blueshift velocities in Table 3 are calculated for each value as the quadratic sum of the error in the centroid estimation from the Gaussian fit and the error associated with the absolute wavelength calibration of the spectra, as explained above. The values followed by an asterisk $(*)$ represent the velocity of the most blueshifted component when a double Gaussian fit was performed for asymmetric profiles. In this case, it is not possible to associate a sensible error with the velocity value as the error from the fit would be largely overestimated. The choice of a double Gaussian profile is in fact arbitrary since the line profile could potentially be fitted as a combination of several Gaussian components at different blueshifted velocities. However, there are no reason a priori to assume more than two profiles and hence a double-Gaussian profile represents the simplest choice.

\subsection{The C4.7 flare}

Figure 6 shows a sequence of EIS monochromatic images as a function of time (from left to right) during the impulsive and peak phases of the $\mathrm{C} 4.7$ class flare. The rasters are numbered from 1 to 5 according to the order of the time intervals indicated by the vertical dashed lines in Fig. 1. The first, second and fourth rows show intensity images formed in the Fe XIV $(\approx 2 \mathrm{MK}), \mathrm{Fe}$ XVI $(\approx 3 \mathrm{MK})$ and Fe XXIII $(\approx 10 \mathrm{MK})$ lines respectively. The last row shows intensity images formed in the blue wing of the Fe XXIII line, which are obtained by integrating the line profile over a wavelength interval corresponding to blueshifts from about $60 \mathrm{~km} \mathrm{~s}^{-1}$ to $230 \mathrm{~km} \mathrm{~s}^{-1} \pm 5 \mathrm{~km} \mathrm{~s}^{-1}$. These maps provide the location of the bluemost component of the line and its evolution over time. The colour scale may change for different rasters in order to show the early and faint hot temperature emission of the Fe XXIII line. The third row shows the Doppler shift velocity of the Fe XVI line, with the colour scale ranging from $-50 \mathrm{~km} \mathrm{~s}^{-1}$ (blue colour) to $+50 \mathrm{~km} \mathrm{~s}^{-1}$ (red colour) for all the rasters. The Fe XVI Doppler maps are calculated by taking the centroid position from the Gaussian fit at each pixel. The Fe XVI rest position was measured for every EIS raster in a background region, as explained in Sect. 3.1. The last column on the right shows AIA images in the 211, 335, and $131 \AA$ filters, taken at the closest time to the EIS Raster 3 (middle column), at around 02:00 UT.

The Fe XXIII emission is first observed in the EIS Raster 2 $(\approx 01: 57$ UT). This early emission is very faint, weakly blueshifted $\left(\approx 30 \mathrm{~km} \mathrm{~s}^{-1}\right)$ and located mainly around the footpoint $\mathrm{K} 2$ and on the spine-like structure. No emission at the footpoint $\mathrm{K} 1$ is observed at this time.

In Raster 3, the Fe XXIII emission is observed to have a blueshift $\left(\approx 100 \mathrm{~km} \mathrm{~s}^{-1}\right)$, as shown in Figs. 7 and 8 . Figure 7 shows the detector images (left) and corresponding spectra (right) of the Fe XXIII spectral window at the footpoints K1 (top panels) and K2 (bottom panels). The spectra were obtained by averaging over 2-3 EIS pixels along the slit direction. The detector images show that the Fe XVI $262.98 \AA$ line is observed all along the EIS slit (as a foreground emission, see Del Zanna et al. 2011a), while the Fe XXIII emission is only observed at the flare footpoints. The detector images are saturated to show the very faint Fe XXIII emission. The Fe XXIII spectrum at K1 (top right panel) shows an asymmetric, but completely blueshifted, broadened, and faint line profile with a centroid position at around $106 \mathrm{~km} \mathrm{~s}^{-1}$. However, very weak more blueshifted components might be also present. The Fe XXIII line profile at K2 (bottom right panel) is more intense and dominated by a line component at rest, with a secondary weaker blue component at around $100 \mathrm{~km} \mathrm{~s}^{-1}$.

A direct comparison between the line profiles at the two footpoints can best be seen in the left panel of Fig. 8, showing the Fe XXIII profiles in K1 (pink) and K2 (blue). The spectra are fitted using the cfit solarsoft routine. The spectrum in $\mathrm{K} 1$ was fitted as a single, completely blueshifted Gaussian 

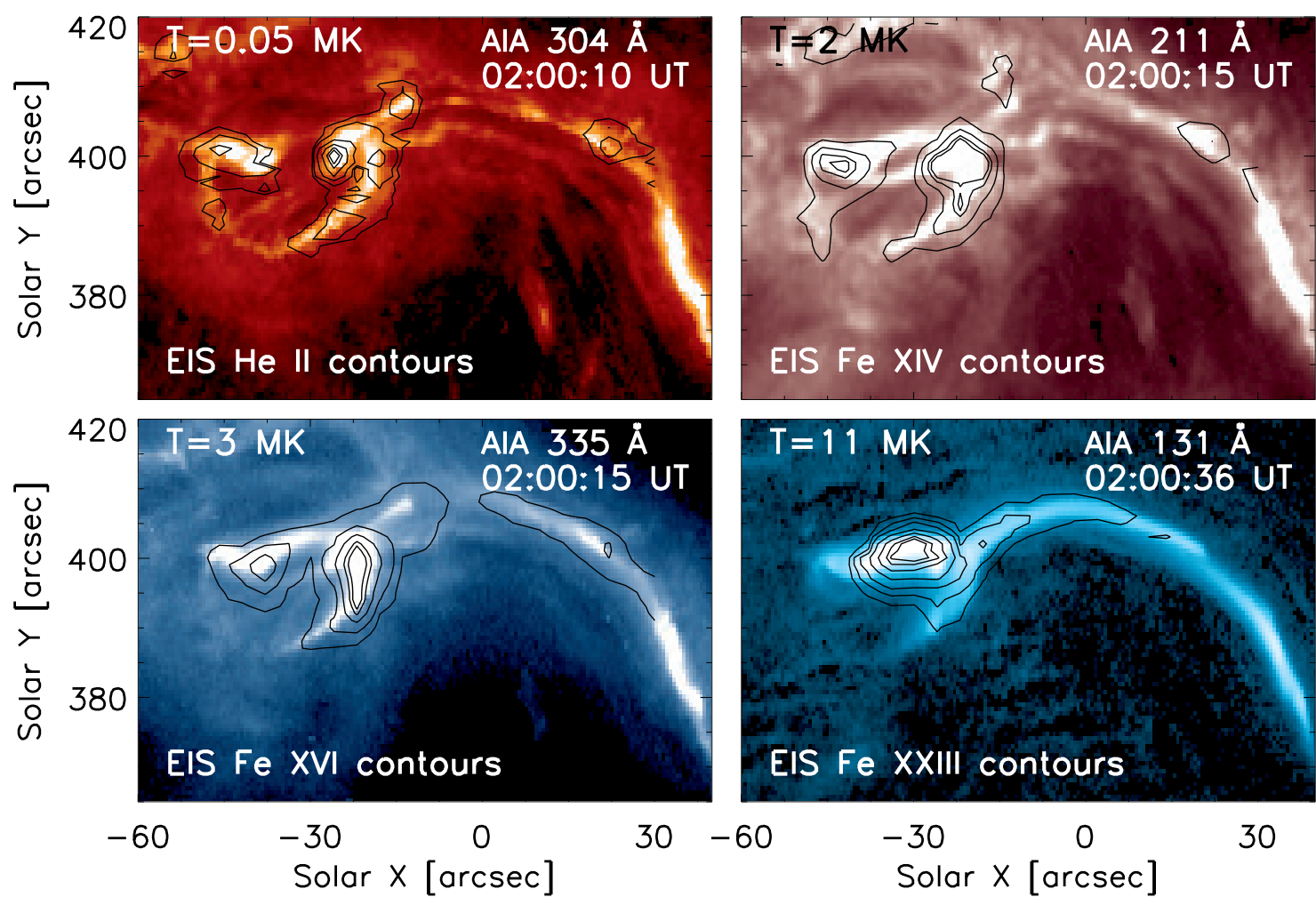

Fig. 4. AIA images in the $304 \AA$ (top left), $211 \AA$ (top right), $335 \AA$ (bottom left), and $131 \AA$ (bottom right) filters for the C4.7 class flare. The field of view of these images is shown in Fig. 3. The EIS intensity contours of the He II, Fe XIV, Fe XVI and Fe XXIII lines, respectively, are overplotted on the AIA images to show the co-alignment between AIA and EIS observations. The temperature of the plasma that dominates the AIA channels is indicated at the top of each image.

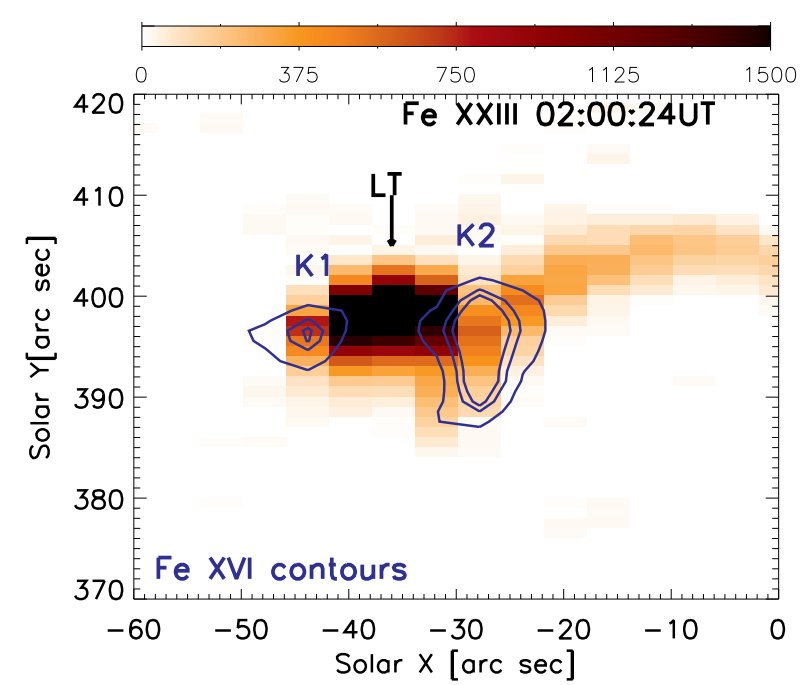

Fig. 5. Zoomed EIS intensity image in the Fe XXIII line with an overplot of the intensity contours of the Fe XVI line (blue). The location of the flare kernels $\mathrm{K} 1$ and $\mathrm{K} 2$ are also indicated in the figure. The flare looptop is indicated as LT.

component, while the asymmetric $\mathrm{K} 2$ profile was fitted by using two Gaussian components, which are indicated by the blue dotted lines. The vertical black line represents the expected rest position of the Fe XXIII line, which was determined as described in Sect. 2.2. The double component profile in $\mathrm{K} 2$ can be interpreted as due to the superposition of the evaporating plasma from the footpoint and the plasma that has already filled the flare loops. The right panel of Fig. 8 shows a Fe XXIII spectrum at the top of
Table 2. EIS spectral lines analysed in this work.

\begin{tabular}{lcc}
\hline \hline Ion & $\begin{array}{c}\text { Wavelength } \\
(\AA)\end{array}$ & $\begin{array}{c}\log T_{\mathrm{m}} \\
(\mathrm{K})\end{array}$ \\
\hline He II & 256.32 & 5.7 \\
Si VII & 274.14 & 5.8 \\
Si VII & 275.35 & 5.8 \\
Fe XIV & 264.79 & 6.3 \\
Fe XIV & 274.20 & 6.3 \\
Fe XVI & 262.98 & 6.4 \\
Fe XXIII & 263.78 & 7.1 \\
\hline
\end{tabular}

Notes. $T_{\mathrm{m}}$ represents the peak of the ion fractional abundance in CHIANTI v.8. The wavelength of the spectral lines are also taken from CHIANTI.

the flare loops (LT, shown in Fig. 5). The line profile at the loop top is at rest and symmetric, confirming our interpretation of the double component profile reported above.

The spectroscopic analysis of the C4.7 class flare suggests that the magnitude of the evaporating flows is around $100-150 \mathrm{~km} \mathrm{~s}^{-1}$ for both flare footpoints. However, the slit is observing the flare footpoints at different times during the EIS raster, and therefore we cannot rule out that higher blueshift velocities were reached, as will be discussed in more detail in Sect. 3.3. Finally, it is interesting to point out that the Fe XVI line is observed to be almost completely blueshifted and symmetric during the impulsive phase $\mathrm{C} 4.7$ class flare with blueshift velocities up to around $40 \mathrm{~km} \mathrm{~s}^{-1}$.

The Fe XXIII blueshifts at the footpoints then decrease in the following rasters, going towards the peak of the flare. 
V. Polito et al.: Analysis and modelling of recurrent solar flares observed with Hinode/EIS on March 9, 2012
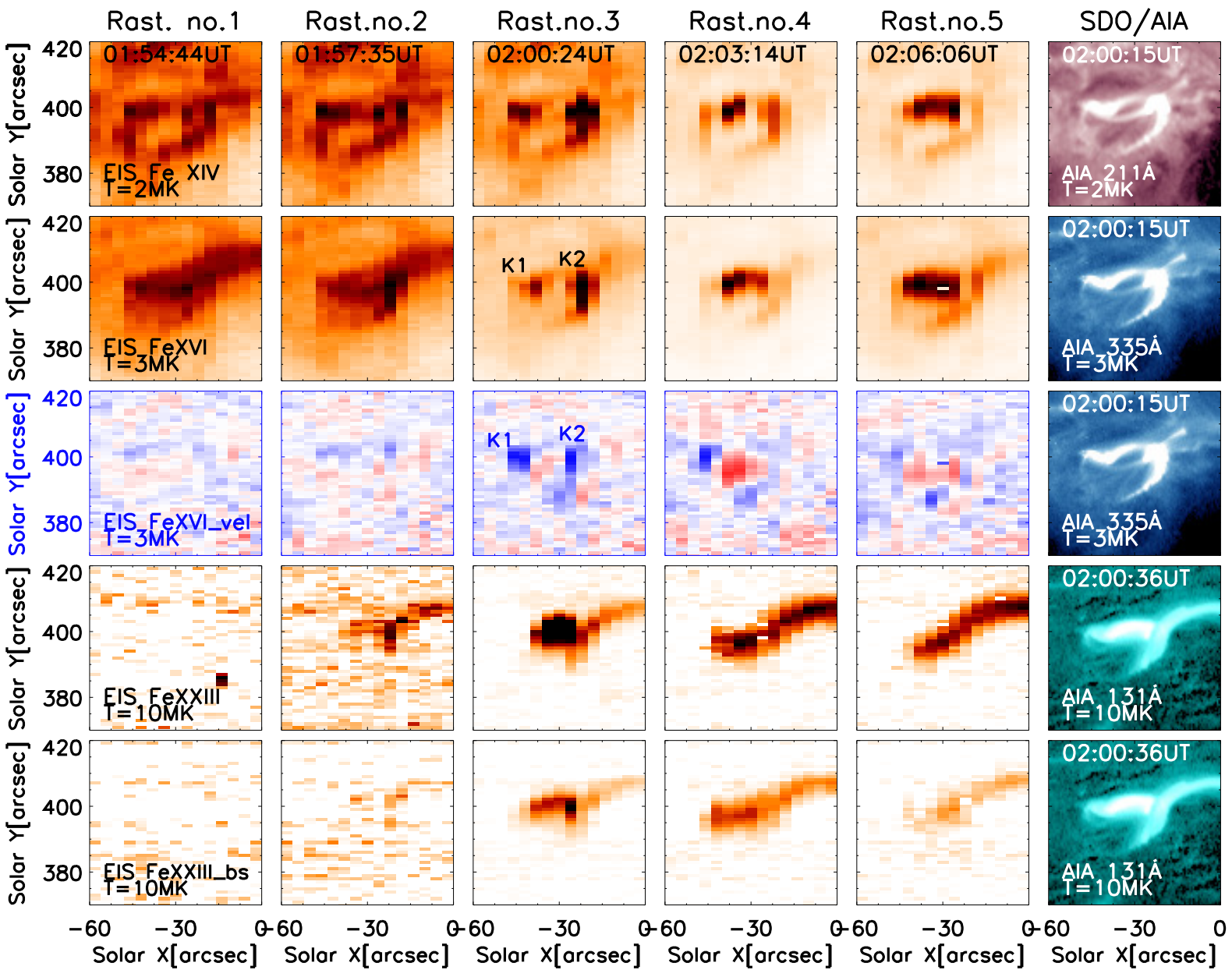

Fig. 6. Sequence of EIS maps showing the Fe XIV intensity (first row), Fe XVI intensity (second row), and Doppler shift (from $-50 \mathrm{~km} \mathrm{~s}{ }^{-1}$ to $+50 \mathrm{~km} \mathrm{~s}^{-1}$, third row), Fe XXIII intensity (fourth row) and Fe XXIII blue wing intensity (fifth row, see text) for different rasters over time (from left to right) for the C4.7 class flare. The last column shows the closest AIA images in the 211, 335, and 131 A filters, to the EIS Raster 3 (middle column). Footpoints K1 and K2 are indicated in the Fe XVI intensity and Doppler shift images in the Raster 3 (third column).

Table 3. Maximum Fe XXIII and Fe XVI upflow velocities during the recurrent flares.

\begin{tabular}{ccccc}
\hline \hline Flare & \multicolumn{2}{c}{$\begin{array}{c}\text { Fe XXIII vel } \\
\left(\mathrm{km} \mathrm{s}^{-1}\right)\end{array}$} & \multicolumn{2}{c}{$\begin{array}{c}\text { Fe XVI vel } \\
\left(\mathrm{km} \mathrm{s}^{-1}\right)\end{array}$} \\
\hline & $\mathrm{K} 1$ & $\mathrm{~K} 2$ & $\mathrm{~K} 1$ & $\mathrm{~K} 2$ \\
C2.0 & $202 \pm 14$ & $60 \pm 7$ & $76^{*}$ & $12 \pm 5$ \\
C4.7 & $146 \pm 10$ & $110^{*}$ & $43 \pm 5$ & $39 \pm 5$ \\
\hline M1.8 (Doschek et al. 2013) & \multicolumn{2}{c}{$150-170$} & \multicolumn{2}{c}{$40-60$} \\
\hline
\end{tabular}

Notes. The values followed by an asterisk ${ }^{(*)}$ represent the velocity of the most blueshifted component when a double Gaussian fit was performed .

In particular, during the Rasters 4 and 5, the EIS images show a strong Fe XXIII emission in the spine-like structure, confirming that this structure is dominated by high temperature $(\gtrsim 11 \mathrm{MK})$ plasma. During these rasters, we can also observe the flare loops that have cooled down and are now visible in the Fe XVI and Fe XIV images, in agreement with the observations reported by Del Zanna et al. (2011a). In addition to the kernels and the spinelike structure, one can also note the presence of a fainter semicircular structure, which is clearly visible in the Fe XIV line (and AIA 211 filter) in Fig. 7.

After 02:10 UT, there is no detectable Fe XXIII emission in the EIS field of view (until the impulsive phase of the following M1 class flare).

\subsection{The C2.0 flare}

Similar to Fig. 6, Fig. 9 shows a sequence of EIS raster images for different emission lines during the impulsive and gradual phases of the C2.0 class flare. The analogy of the global evolution obtained comparing Figs. 6 and 9 confirms the homologous nature of the flares.

During the first EIS raster (at around 01:23 UT), there is no significant Fe XXIII emission observed at the flare footpoints. From around 01:26 UT (Raster 2 in the figure), some faint Fe XXIII emission is observed in both footpoints. The detector images and corresponding Fe XXIII and Fe XVI spectra at these locations are shown in Fig. 10. The top panel shows that there 

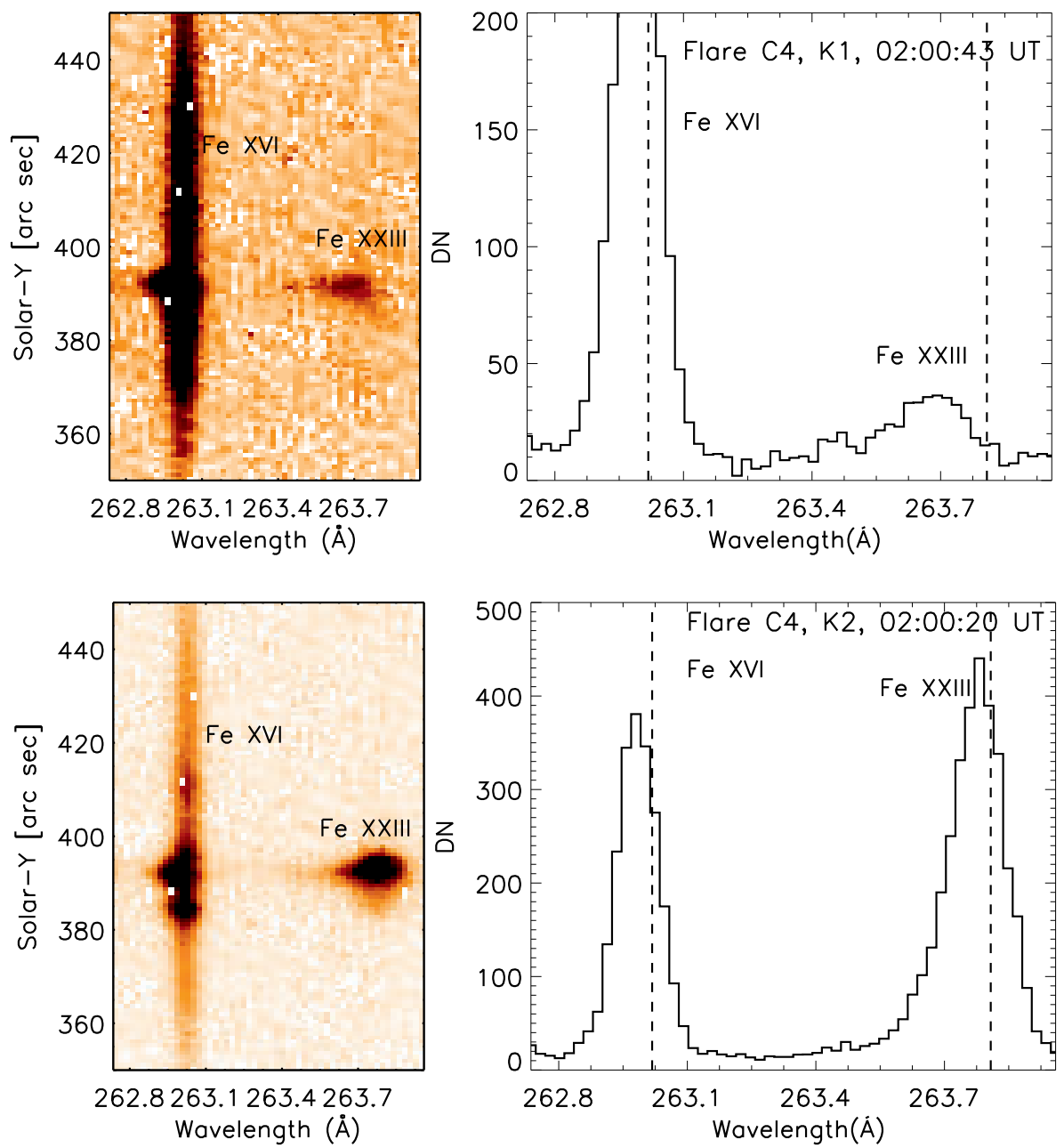

Fig. 7. Left panels: CCD detector images at the slit position corresponding to the footpoints K1 (top left) and K2 (bottom left) in Fig. 5. The Fe XVI and Fe XXIII spectral lines are indicated in each image. Right panels: spectra of the Fe XXIII EIS window at the K1 (top right) and K2 (bottom right) footpoints, obtained by averaging over few pixels where the Fe XXIII emission is most visible in the corresponding CCD images on the left.
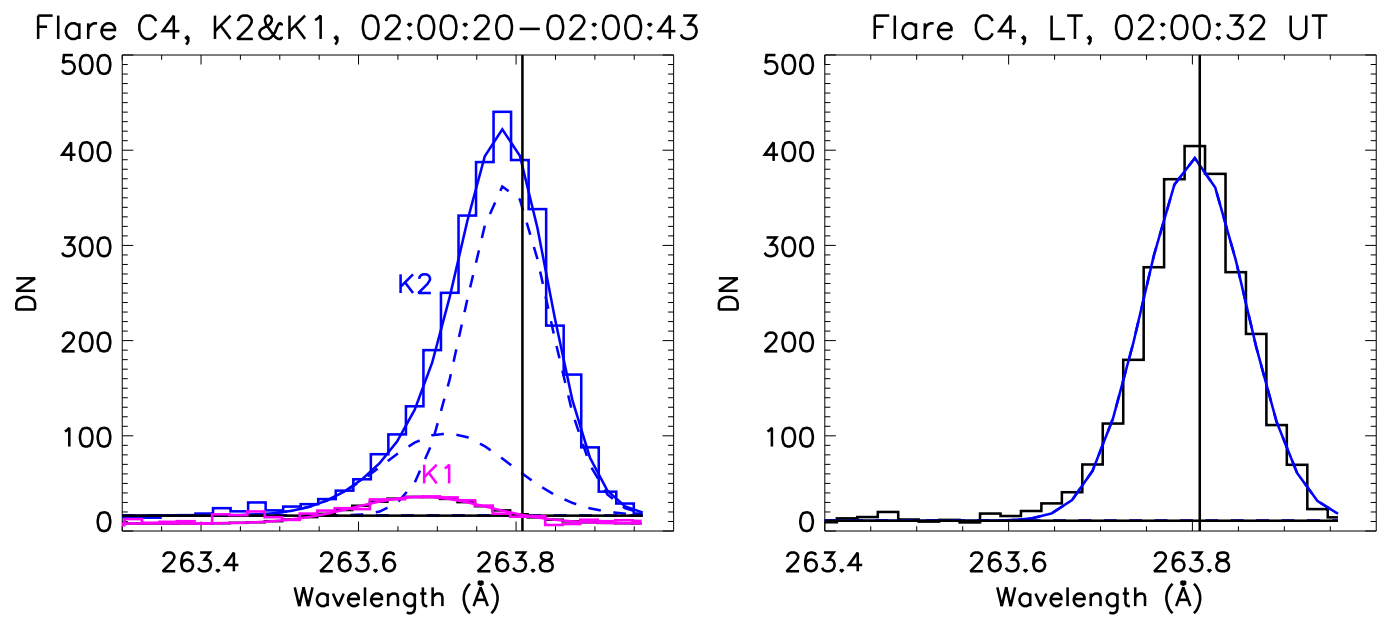

Fig. 8. Left panel: Fe XXIII spectra at the footpoint K1 (pink histogram lines) and K2 (blue histogram line). The blue dashed lines represent the double Gaussian components of the fit and the blue continuous line is the superposition of the two components. Right panel: Fe XXIII spectrum (black histogram line) at the flare loop top (LT, see Fig. 5). The continuous line represents the Gaussian fit. The vertical black lines in the left and right panels indicate the expected rest position of the Fe XXIII line.

is a very faint high temperature Fe XXIII emission in the very early impulsive phase at the footpoint $\mathrm{K} 1$. This emission is completely blueshifted by around $200 \mathrm{~km} \mathrm{~s}^{-1}$, which is the highest velocity recorded in this observation. In contrast, at footpoint K2 (bottom panel) the Fe XXIII profile is blueshifted by only around $60 \mathrm{~km} \mathrm{~s}^{-1}$. The analysis of the C2.0 class flare blueshifts seems therefore to suggest that the Fe XXIII upflows at footpoint K1 are larger than at footpoint $\mathrm{K} 2$. However, it is important to bear in 

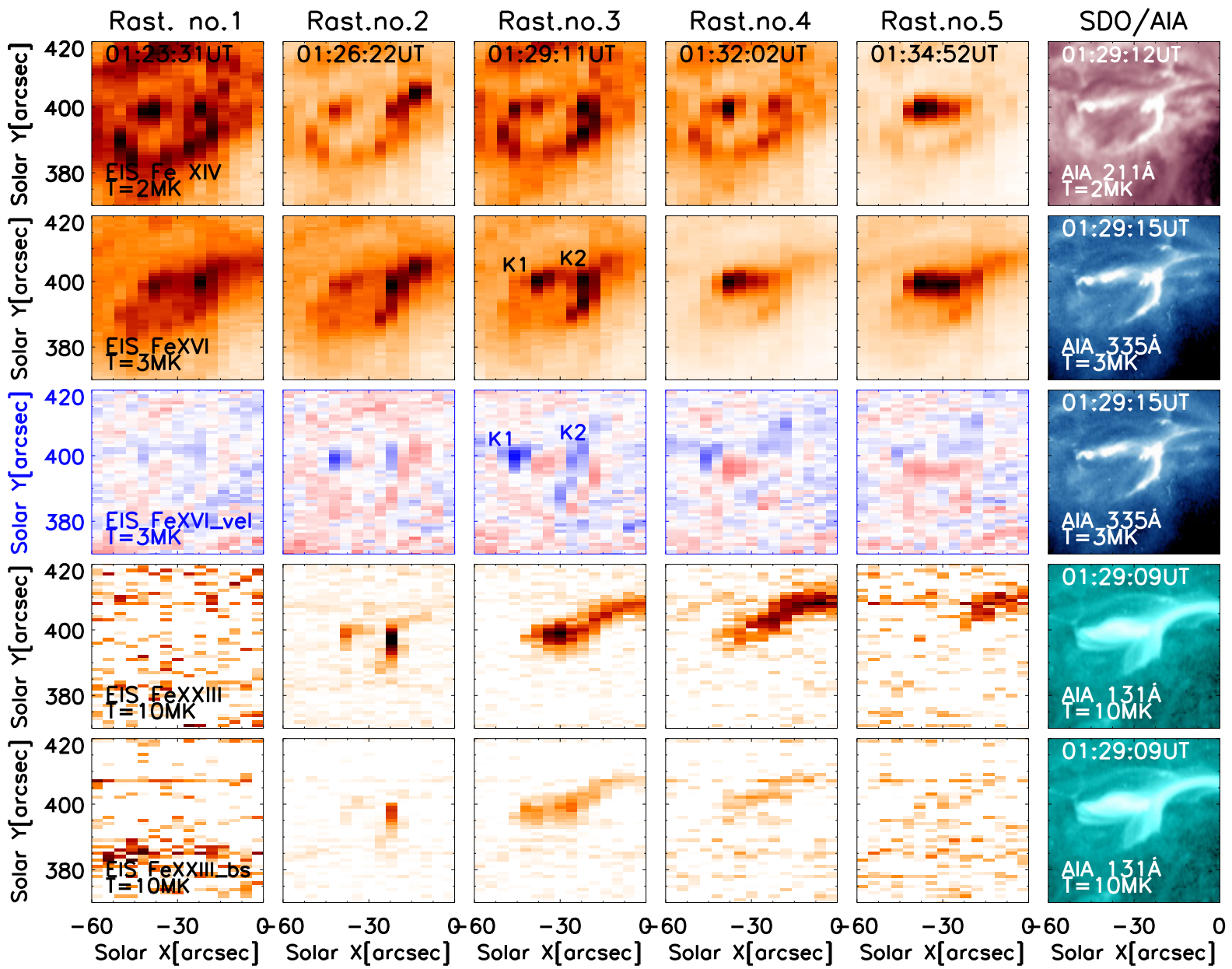

Fig. 9. Similar to Fig. 6 for the $\mathrm{C} 2.0$ class flare.

mind that the difference between the upflows at the two footpoints might also be related to the fact that the EIS slit is rastering over these locations at different times.

In the following raster, the blueshift velocity decreases to around $100 \mathrm{~km} \mathrm{~s}^{-1}$ at the $\mathrm{K} 1$ footpoint, while it remains at $\approx 60$ $\mathrm{km} \mathrm{s}^{-1}$ at footpoint $\mathrm{K} 2$. There are then no significant upflows in the Fe XXIII line after about 01:30 UT and the Fe XXIII emission is not visible after $\approx 01: 36 \mathrm{UT}$. The strongest blueshift in the Fe XVI line is also observed at the footpoint $\mathrm{K} 1$, as shown in the spectrum in Fig. 11. This is the only case we observed where the Fe XVI line profile shows a significant asymmetry. The line was fitted with two Gaussian components (blue dotted lines), the most blueshifted one showing an upflow velocity of $\approx 76 \mathrm{~km} \mathrm{~s}^{-1}$.

The semi-circular feature observed in the $\mathrm{C} 4.7$ flare is also clearly visible here, especially in the Fe XIV line. Such a structure, together with the spine-like brightening discussed above, is suggestive of a circular ribbon flare that is usually generated by reconnection at a null point of the magnetic field at coronal heights, see e.g. Masson et al. (2009), Reid et al. (2012), Sun et al. (2013) and references therein. However, the confirmation of the presence of such a topological feature and its role in the homologous nature of the flares studied requires more information on the underlying magnetic field (for further discussion see Sect. 6).

From a comparison of the blueshifts in the two C-class flares, it seems that higher velocities are reached during the smaller C2.0 class flare. However, we emphasize that the timing of the observations should also be taken into account. For the C4.7 class flare, the area around the footpoint K1 was first observed by EIS at around 01:58 UT, that is $\approx 177 \mathrm{~s}$ after the beginning of the flare as measured by the GOES satellite (second column in Table 1). No significant Fe XXIII is observed there at that time. During the following raster, EIS observes a $\approx 145 \mathrm{~km} \mathrm{~s}^{-1}$ blueshift at this footpoint at around 02:00 UT, that is already $\approx 300 \mathrm{~s}$ after the beginning of the impulsive phase. For the $\mathrm{C} 2.0$ class flare, the highest blueshift of $\approx 200 \mathrm{~km} \mathrm{~s}^{-1}$ is observed at around $220 \mathrm{~s}$ into the impulsive phase of the flare. This suggests that larger upflows at K1 might have been present during the impulsive phase of the C4.7 class flare between the EIS Rasters 2 and 3 in Fig. 6, but were missed by the spectrometer.

\section{Electron number density from EIS observations}

During flares, the ratio of the Fe XIV 264.79 and $274.20 \AA$ lines observed by EIS provides useful electron number density diagnostics for the $\approx 2$ MK plasma in the interval $\approx 10^{9}-10^{11} \mathrm{~cm}^{-3}$ (e.g. Del Zanna et al. 2011a; Brosius 2013; Polito et al. 2016). It is generally assumed that the Fe XIV line at $264.79 \AA$ is free from significant blends in active region and flare spectra, as discussed by Del Zanna et al. (2006). In contrast, the Fe XIV $274.20 \AA$ is known to be blended with a Si VII line at $274.175 \AA$. The contribution of this latter spectral line can be estimated by measuring the intensity of the other Si VII line observed by EIS (at $275.35 \AA$ ), which forms a branching ratio with the 274.175 $\AA \mathrm{Si}$ VII line. The contribution of the Si VII to the 

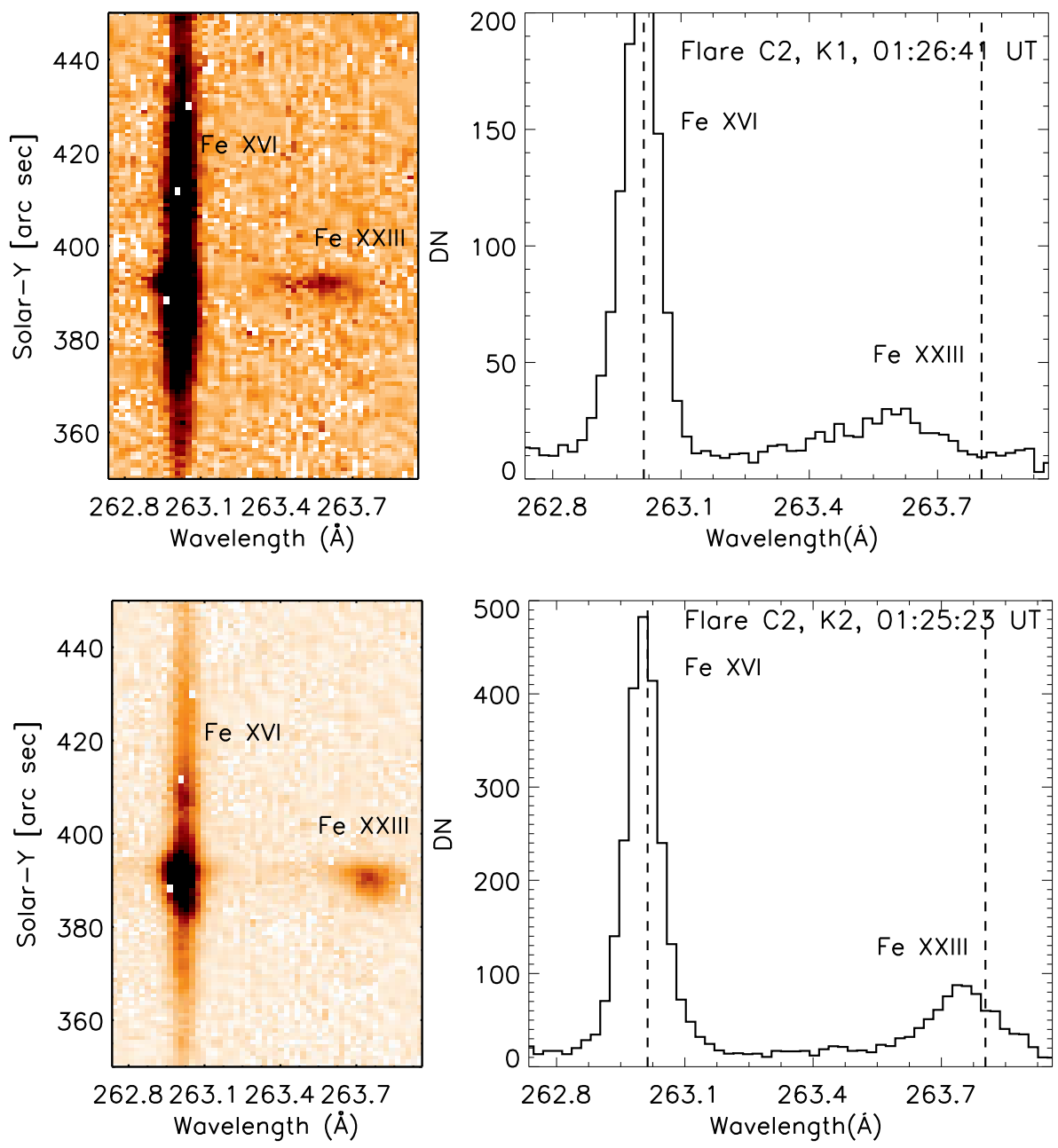

Fig. 10. Similar to Fig. 7 for the C2.0 class flare.

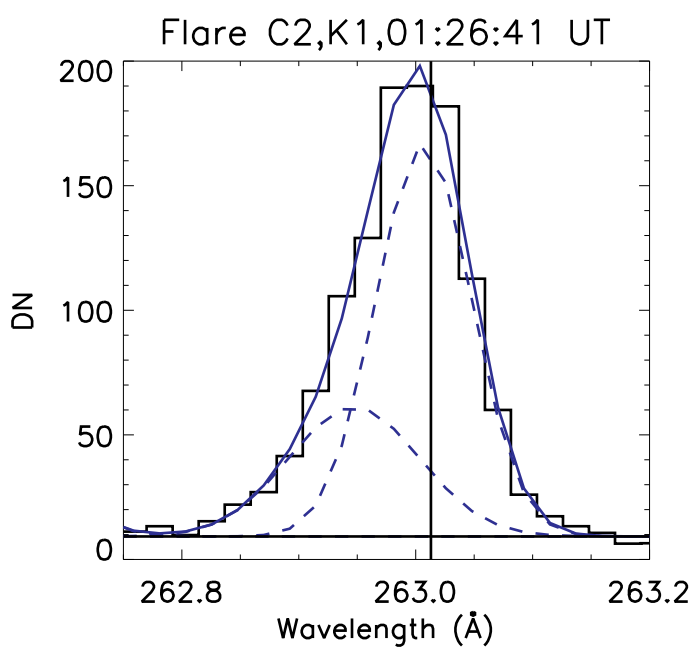

Fig. 11. Fe XVI spectrum at the footpoint K1 during the impulsive phase of the $\mathrm{C} 2.0$ class flare. The line profile is asymmetric and was fitted with two Gaussian components, which are indicated by the blue dashed lines.

Fe XIV line around $274.2 \AA$ however is usually estimated to be very small, of the order of $4 \%$ (see e.g. Del Zanna et al. 2011a; Brosius 2013). Del Zanna et al. (2011a) obtained Fe XIV densities from rest (foreground) and blue components separately in their analysis of a B-class flare. The rest component densities

were in agreement with the averaged AR densities, while the blueshifted component densities reached values near the high density limit $\left(\approx 10^{11} \mathrm{~cm}^{-3}\right)$. In this work, we do not observe any clear asymmetry in the Fe XIV line profiles which were thus fitted as a single Gaussian component, as in Polito et al. (2016). However, the Fe XIV lines at the footpoints are observed to be broadened and, hence, we cannot rule out that different line components are present within the spectral resolution of the instruments. Our estimates are therefore likely to represent average density values.

We measured the ratio of the FeXIV 264.79 and $274.20 \AA$ lines (after removing the Si VII contribution) from the impulsive to the gradual phase for the three confined flares and obtained the electron number density by using Fe XIV atomic data from the CHIANTI v8 database. Figure 12 shows the density maps (expressed as $\mathrm{cm}^{-3}$ and in a logarithmic scale) over time for the C4.7 class flare. We can observe that the flare footpoints and ribbons exhibit a higher electron density compared to the surrounding AR, with values ranging around $10^{10.2} \mathrm{~cm}^{-3}$ during the evaporation phase (at around 01:57 UT) and gradually increasing up to the high density limit of $10^{11} \mathrm{~cm}^{-3}$ at around 02:03 UT, just after the peak of the flare. Since the Fe XIV line ratio reaches the high density limit, we cannot rule out that the plasma density there is above $10^{11} \mathrm{~cm}^{-3}$. This is also consistent with the density diagnostics reported by Doschek et al. (2013), who showed that the Fe XIV line ratio reaches the high density limit of $10^{11} \mathrm{~cm}^{-3}$ during the M-class flare. 


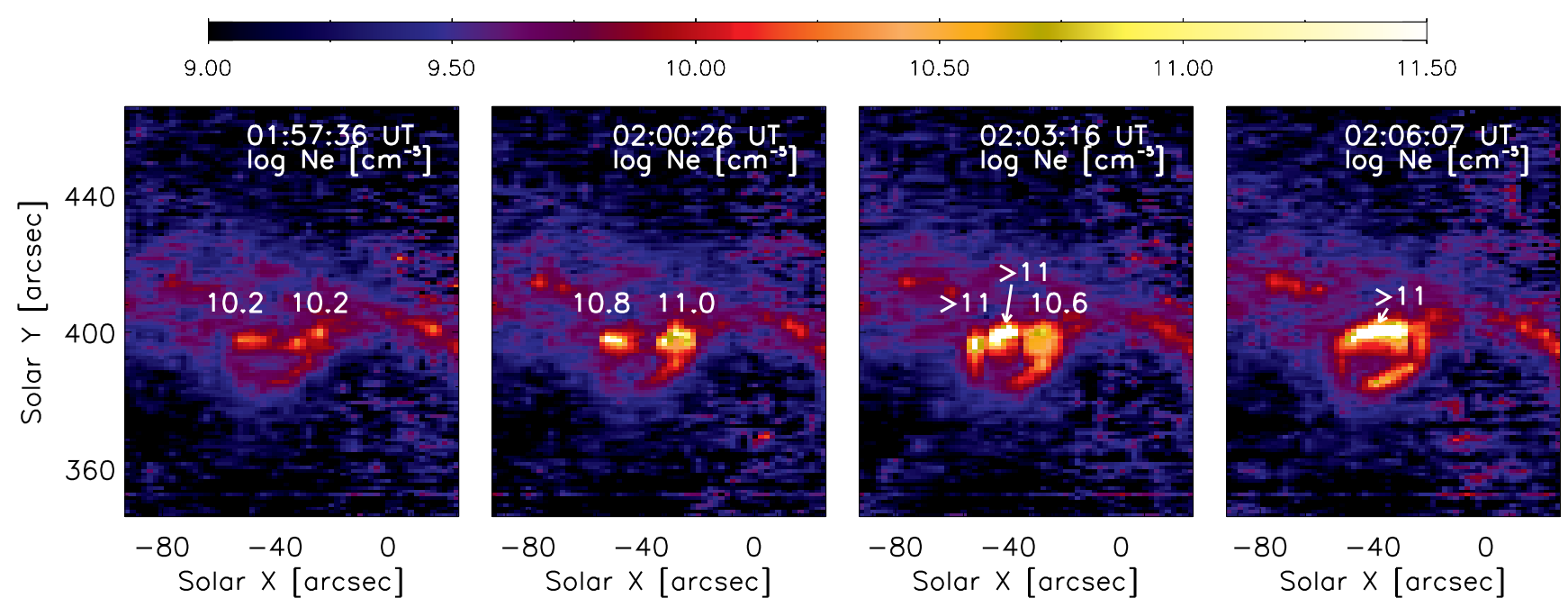

Fig. 12. Density maps (in a log scale) for different times during the $C 4.7$ class flare. The maps were obtained by using the ratio of the Fe XIV lines observed by EIS and atomic data from CHIANTI v8. The average density values measured at the flare footpoints are indicated in each panel.
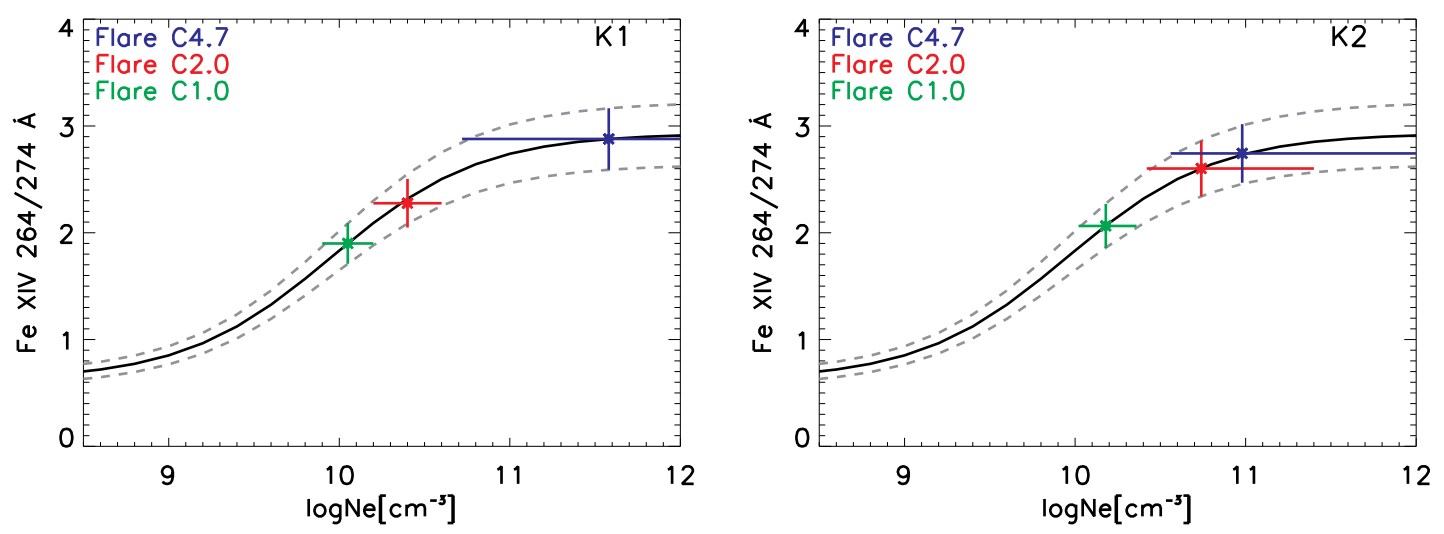

Fig. 13. Maximum density estimates based on the measurements of the Fe XIV 264/274 A ratio during the three C-class flares, as indicated by different coloured points and associated error bars (as described in the legend on each image) for the footpoints K1 (left panel) and K2 (right panel). The solid black curve indicates the theoretical ratio calculated using CHIANTI v8 atomic database. The gray dotted curves represent a $\pm 10 \%$ error in the theoretical ratio.

Table 4. Peak $\log N_{\mathrm{e}}$ for the three C-class flares. See text for discussion of minimum and maximum values.

\begin{tabular}{ccc}
\hline \hline Flare & \multicolumn{2}{c}{$\begin{array}{c}\text { Log } N_{\mathrm{e}}(\min , \max ) \\
\mathrm{cm}^{-3}\end{array}$} \\
\hline & $\mathrm{K} 1$ & $\mathrm{~K} 2$ \\
C1.0 & $10.0(9.9,10.2)$ & $10.2(10.0,10.4)$ \\
C2.0 & $10.4(10.2,10.6)$ & $10.7(10.4,>11.0)$ \\
C4.7 & $>11.0(10.7,>11.0)$ & $11.0(10.6,>11.0)$ \\
\hline M1.0 (Doschek et al. 2013) & \multicolumn{2}{c}{$>11.0$} \\
\hline
\end{tabular}

The density maps for the $\mathrm{C} 2.0$ and $\mathrm{C} 1.0$ class flare are reported in Appendix A, and the values of maximum density observed at the footpoints of these three flares are summarized in Table 4. The absolute errors associated with these density estimates should include the uncertainty in the atomic data from CHIANTI, as well as the error on the observed line ratio from EIS, also taking the contribution of the Si VII blend into account.

The uncertainty associated with the individual Fe XIV line intensity is due to the error in the radiometric calibration and the error associated with the Gaussian fit; this latter error is very small, that is, around few percent of the intensity values.
However, the uncertainty in the atomic data and radiometric calibration of the EIS throughputs affects the density measured during the three flares in the same way. In this work, we are interested in comparing density measurements obtained in different flares and therefore we consider only the sources of error that are not systematic, that is, the error associated with the Fe XIV line ratios for the three observations. A possible approach is to estimate how the uncertainty in the line ratio can affect the density diagnostics, as shown in Fig. 13. In this figure, a $\pm 10 \%$ maximum error bar for the line ratios (given by the errors associated with the Gaussian fit and Si vII blend) is plotted on the $y$-axis 
for the three C-class flares. This error is then propagated on the $x$-axis (density space), assuming the same theoretical ratio from CHIANTI (solid curve) for all the density measurements. This method gives a density interval associated with each measured line ratio, which is reported in the curved brackets next to each value of density in Table 4, providing an estimate of the relative error in the density values for the three flares. Figure 13 shows that this error is smaller if the density measured is well within the density sensitivity of the Fe XIV line ratio (i.e. from $\approx 10^{9.5}$ to less than $10^{11} \mathrm{~cm}^{-3}$ ), but this error becomes larger if the density measured is at the edge of the density sensitivity of the line ratio (around $10^{11} \mathrm{~cm}^{-3}$ ). In particular, in this latter case, only a lower value of uncertainty can be estimated, because the upper limit of the line ratio will be outside the density sensitivity limit of the line ratio; however, the upper limit is still consistent with a density above or close to $10^{11} \mathrm{~cm}^{-3}$ considering a $10 \%$ uncertainty in the atomic data (shown with gray lines in Fig. 13). Within the estimated errors, the maximum electron number densities observed during the $\mathrm{C} 1.0$ class flare are lower than the C2.0 and $\mathrm{C} 4.7$ flares for both footpoints $\mathrm{K} 1$ and $\mathrm{K} 2$, while these latter flares reach in principle similar values of density at the footpoint K2. However, by comparing the density maps in Figs. 12, A.2, and A.1, one can observe that higher densities (above $10^{10} \mathrm{~cm}^{-3}$ ) are reached in the $\mathrm{C} 4.7$ class flare at both footpoints for a longer period of time during the impulsive and peak phase of the flare (note that the three figures have the same colour scale).

\section{Plasma temperature from AIA observations}

Despite the multi-thermal nature of the AIA channels, during flares the $94 \AA$ and $131 \AA$ AIA channels are dominated by emission from Fe XVIII (formed at $\sim 7 \mathrm{MK}$ ) and Fe XXI (formed at $\sim 11$ MK) respectively (O'Dwyer et al. 2010; Boerner et al. 2014). It has been shown previously that the intensity ratio of these bands can be used to provide reliable temperature diagnostics for the flaring plasma (see e.g. Petkaki et al. 2012; Del Zanna \& Woods 2013). The AIA filter ratio method relies on the assumption that the plasma is isothermal and dominated by plasma formed in the temperature sensitivity range of the AIA 131 and $94 \AA$ ratio (8-12 MK). This is a sensible assumption close to or during the peak of flares. In fact, Figs. 6 and 9 show that during the peak of the flares the flare loops are dominated by emission from the Fe XXIII ion (formed at around $10 \mathrm{MK}$ ), while no emission is observed at that time from cooler emission lines (e.g. Fe XVI formed at $3 \mathrm{MK}$ and Fe XIV formed at $2 \mathrm{MK}$ ).

In Fig. 14, we show the evolution of the plasma temperature for the three C-class flares using the 131 and $94 \AA$ filter ratio. The AIA images were badly saturated during the peak phase of the C4.7 and C2.0 class flares and cannot be used for diagnosing the temperature. The first three panels of Fig. 14 show the temperature maps for the $\mathrm{C} 4.7, \mathrm{C} 2.0$ and $\mathrm{C} 1.0$ class flares, respectively from top to bottom. These maps were calculated $2 \mathrm{~min}$ and $3 \mathrm{~s}$ before the peak of each flare, which is the closest time to their respective peaks in which the AIA images for each of the three flares are not saturated. The bottom panel shows the temperature during the peak of the smallest C1.0 flare, in which the AIA images were not significantly saturated. The first three panels of Fig. 14 allow us to compare the plasma temperature at the same time into the evolution of the three flares. Two minutes before the peak, the largest $\mathrm{C} 4.7$ class flare reaches higher temperatures (up to $\log T[\mathrm{~K}] \approx 7$ ) than the smaller $\mathrm{C} 2.0$ and $\mathrm{C} 1.0$ class flares. The highest temperatures during the $\mathrm{C} 4.7$ flare are observed along the flare ribbons and spine-like structure, confirming that this latter
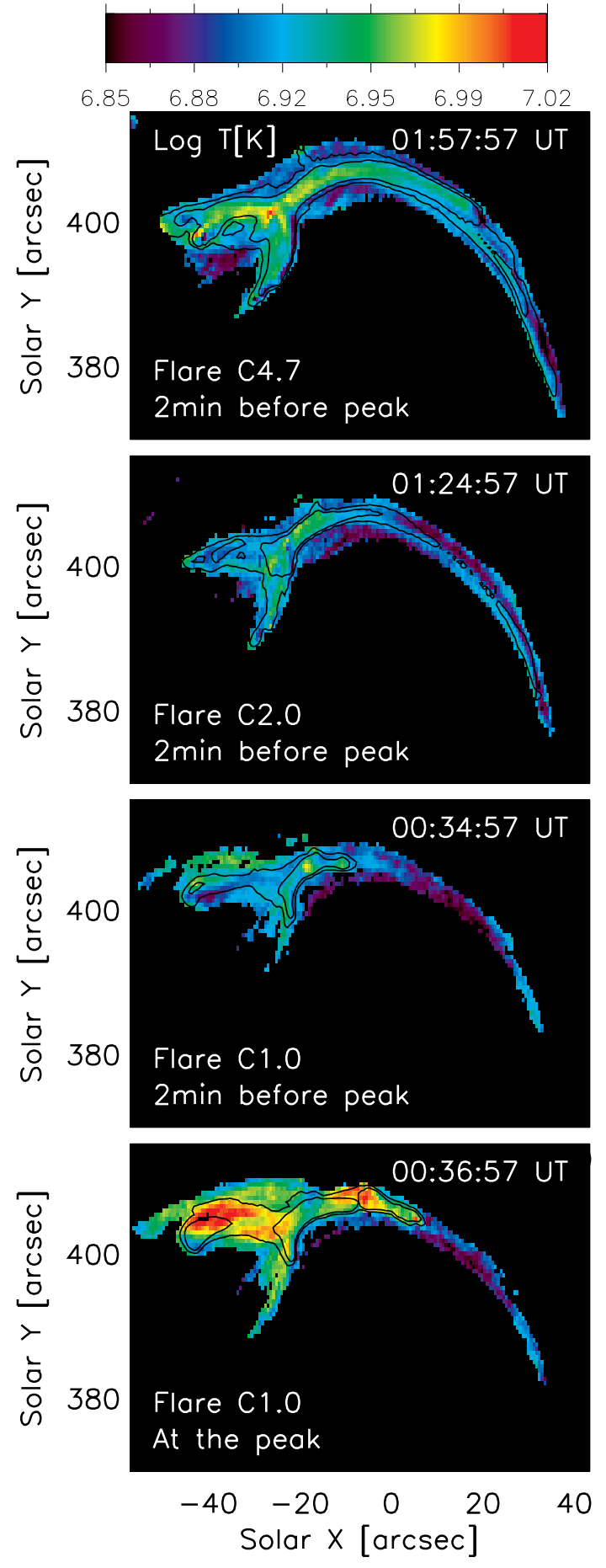

Fig. 14. Temperature maps (in Log scale) of the three C-class flares obtained from the 131 and $94 \AA$ AIA filter ratio two minutes before the peak time for each flare $(\mathrm{C} 4.7, \mathrm{C} 2.0$ and $\mathrm{C} 1.0)$ and at the peak of the C1.0 flare. The intensity contours of the $131 \AA$ channel are overplotted in each panel.

feature is dominated by hot plasma. Moreover, Fig. 14 shows that a high temperature (up to around $10 \mathrm{MK}$ ) is also reached during the small C1.0 class flare, although only during the peak of the flare.

Assigning an error to the absolute temperature diagnostics is not straightforward. The largest uncertainty is given by the error in the calibration of the SDO/AIA filters, which was estimated to be $\approx 25 \%$, including the degradation of the filter responses 
(Boerner et al. 2014). This uncertainty affects the absolute values of temperature shown in Fig. 14, but we can still make a reliable comparison between the relative temperatures during the three flares, since the diagnostics are affected by the same calibration error. Excluding the uncertainty in the filter calibration, the relative values of temperature are then mainly affected by the error in the total counts measured in the 131 and $94 \AA$ filters, which is given by the standard deviation, i.e. the square root of the total counts. We estimated this error to result in a relative uncertainty of only around $1-2 \%$ for the temperature values shown in Fig. 14.

\section{Analysis of the magnetic field structure during the recurrent flares}

\subsection{NLFFF extrapolation}

The HMI instrument (Scherrer et al. 2012) provides full disk vector magnetograms at $0.5^{\prime \prime}$ pixel size and 12 min cadence. The vector magnetogram of AR11429 at 01:12UT of March 9, 2012, is included in the patch 1449 of the HARP catalogue ${ }^{1}$. The standard SHARP data products are treated for the removal of the ambiguity in the direction of the transverse field and cylindrical equal area projection remapping (see Hoeksema et al. 2014, for more details). The area of interest was extracted from the HARP patch data, the resolution was halved using a fluxconserving coarsening, and a median smoothing with a 7-pixel boxcar was applied to all three field components to reduce smallscale fluctuations.

The compatibility of the vector magnetogram as a boundary condition for the NLFFF extrapolation can be improved by preprocessing the magnetogram to reduce Lorentz forces (see e.g. Schrijver et al. 2008). For that purpose, we employed the technique by Fuhrmann et al. (2007), which allows one to fix a limit to the modifications of each observed component separately. In particular, in the present application, preprocessing was only applied to the horizontal components. The maximum variation of these measured values was constrained-in each pixel-by the largest value between $50 \mathrm{G}$ and $30 \%$ of the local value. These maximal ranges of variation resulted in an average modification of $45 \mathrm{G}$ (respectively, $51 \mathrm{G}$ ) in the $B_{x}$ (respectively, $B_{y}$ ) component, and in a decrease of the total Lorentz force on the magnetogram from 0.13 before preprocessing to 0.03 after preprocessing, according to the definition used in Metcalf et al. (2008).

The preprocessed vector magnetogram was then extrapolated to build the coronal field model using the implementation of the magneto-frictional method described in Valori et al. (2010) with open lateral and top boundaries and on three levels of successive grid refinement. The resulting numerical model has a fraction of current perpendicular to the magnetic field in the volume equal to 0.4 . This is concentrated in the lower volume directly connected to the residual Lorentz forces in the magnetogram: indeed, the horizontal plane-average fraction of the current density perpendicular to the field drops with height to $2 \%$ within the first 15 pixels. The relatively high forces are due partly to the system being between dynamical phases (i.e. the flare C1.0 flare at 00:34UT and the C2.0 flare at 01:23UT, see Table 1), but also to the very limited preprocessing applied to the magnetogram. Despite the limited preprocessing, however, the fraction of the magnetic energy associated with errors in the solenoidal

\footnotetext{
1 http://jsoc.stanford.edu/doc/data/hmi/harp/harp_ definitive/2012/03/09/
}

property, and quantified by applying the method in Valori et al. (2013), is limited to $4 \%$.

Because of the presence of non-zero field divergences and Lorentz forces, the NLFFF extrapolations must be compared with observational proxies of the magnetic configuration. In particular, an approximate alignment was performed using the line of sight magnetogram from the HMI instrument to compare AIA observations with the NLFFF extrapolation. When included in the 3D extrapolated volume, the HMI-image plane was rotated so as to be tangent to the centre of the field of view of the vector magnetogram at the bottom of the extrapolation data cube. Next, the image was stretched and scaled until the PIL of the line of sight magnetogram coincided with the PIL of the vertical field of the vector magnetogram. The geometrical transformation of the line of sight magnetogram obtained in this way was then applied to all AIA images. Such a procedure is necessarily approximate in many respects, not least because of the different heights at which the line of sight magnetogram and AIA images are displayed in the observer's projection. Therefore, such comparisons can only be considered to be qualitative.

The underlying topology of the NLFFF extrapolation is most efficiently studied using the distribution of the quasiseparatrix layers (see e.g. Demoulin et al. 1996), which represent volumes of sharp gradients in the field line connectivity. The connectivity gradient is quantified by the squashing degree $Q$ (Titov et al. 2002), and is computed here using the method in Pariat \& Démoulin (2012). In the photospheric $Q$-map obtained in this way, high values of $Q$ correspond to separations between different areas of connectivity.

\subsection{Magnetic field analysis}

By comparing some selected field lines from the extrapolation with simultaneous (at $\approx 01: 12$ UT) EUV images from AIA, one can readily recognize the elongated filament corresponding to the sheared and twisted flux system along the PIL (see Fig. 15). The AIA images and the line of sight magnetogram from SDO/HMI in Fig. 15 are on the (observer) image plane, whereas the vector magnetogram used for the NLFFF extrapolation was re-mapped to a Cartesian grid using a CEA projection (see Sect. 6.1). A careful comparison between the middle panel of Fig. 15 and the online AIA movie also shows a good match between individual dark strands forming the filament, and individual sections of the flux rope/sheared structure above the PIL in the NLFFF extrapolation (for instance, the brown southern field lines, or the core group of red/violet/blue field lines in Fig. 15). In addition, the elongated spine-like structure in the core, which is already recognizable at this time in the top panel of Fig. 15, has a good correspondence with the spine-like blue field lines bundle in the extrapolation (see below for the identification of the associated blue fan-like field lines at its eastern end). Such details corroborate the quality of the extrapolation despite the limitations discussed in Sect. 6.1.

The magnetic helicity of the NLFFF extrapolation, which was computed following the method in Valori et al. (2012), is $-2.7 \times 10^{43} \mathrm{Mx}^{2}$ (corresponding to 0.05 in units of flux squared). Estimations of the helicity of the same active region (but for $24 \mathrm{~h}$ before) were performed by Patsourakos et al. (2016) using three different methods, with values ranging from $-0.4 \times 10^{43} \mathrm{Mx}^{2}$ to $-3.3 \times 10^{43} \mathrm{Mx}^{2}$. The value obtained with a NLFFF extrapolation comparable with ours (Chintzoglou et al. 2015) is $-0.8 \times 10^{43} \mathrm{Mx}^{2}$, which is consistent with our value considering the time span between the two extrapolations. 

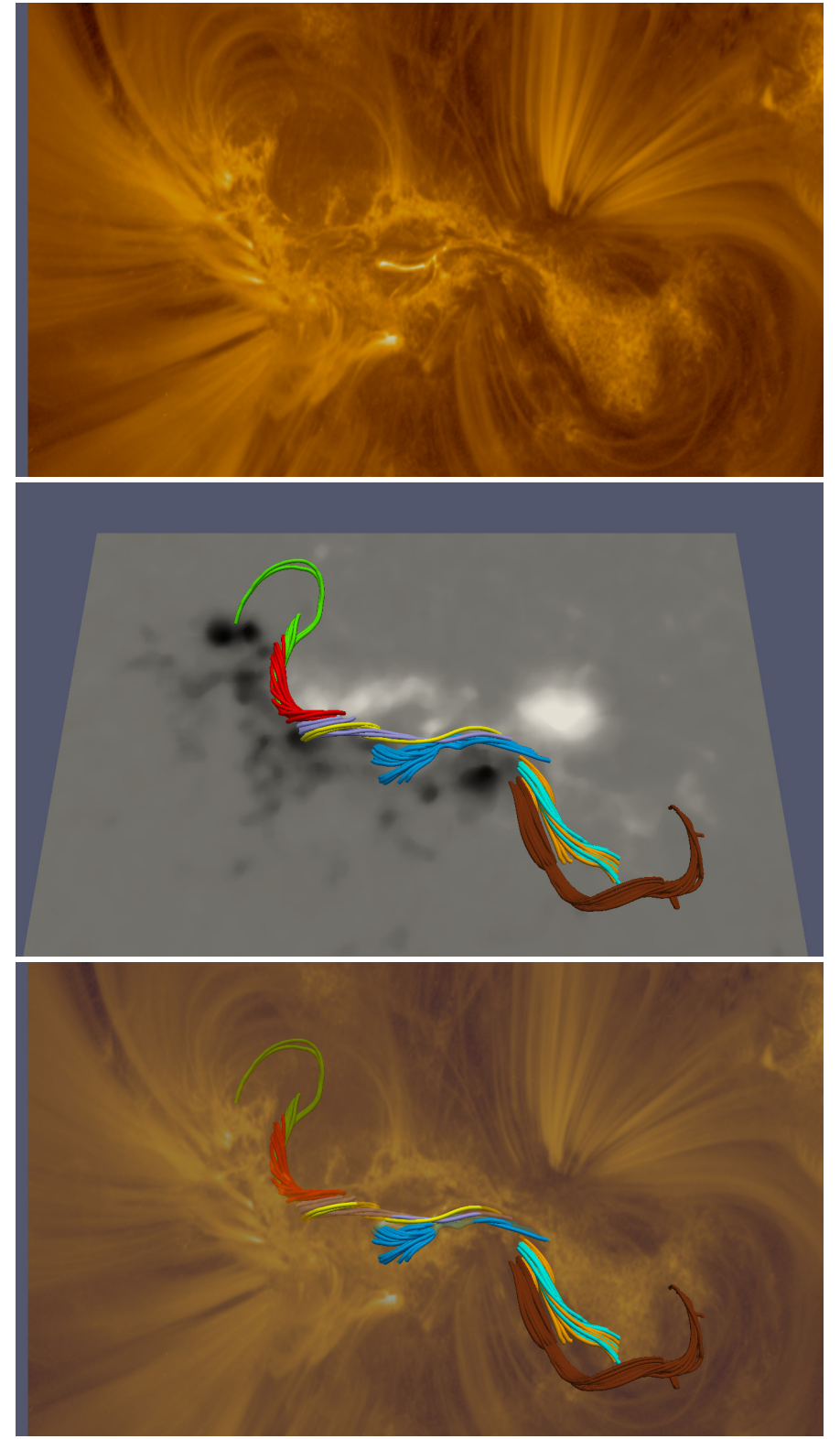

Fig. 15. Comparison between NLFFF extrapolation and AIA $171 \AA$ image from the AIA viewpoint, at around 01:12 UT. Top: AIA $171 \AA$ image. Middle: NLFFF extrapolation, where different colors represent different sections of the flux rope. Bottom: overlay of the AIA $171 \AA$ Amage and the same field lines as in the middle panel.

The free energy, estimated as in Valori et al. (2013), is 27.4\% of the total magnetic energy, which is a value almost seven times larger than the error associated with a violation of the solenoidal property in the field. Therefore, the extrapolation shows beyond doubt that the AR under study had significantly high values of both free energy and helicity, and was thus in a non-potential state. Next, we can use the NLFFF extrapolation at 01:12UT to interpret some of the observations at a later time, on the grounds that the configuration is not changing radically in the following 48 minutes preceding the C2.0 flare (as shown by the AIA Movie 1 and Fig. 2). In particular, we search for an interpretation of the semi-circular and spine-like features which are simultaneously illuminated during all the flares; an example is shown in Fig. 16.
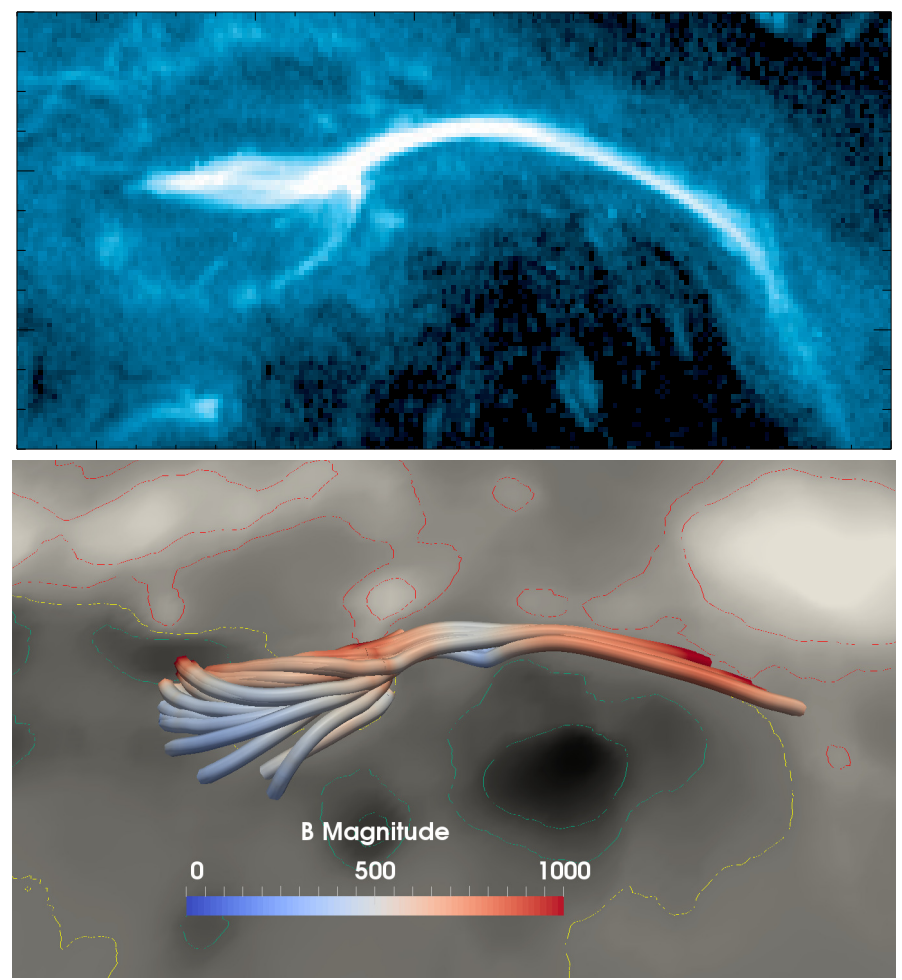

Fig. 16. Comparison between the AIA $131 \AA$ image at around 01:12 UT, zoom on the ribbon region (top), and selected field lines from the NLFFF extrapolation (bottom). The colour coding in the latter represents the magnetic field strength.

First, using a null finder algorithm (Démoulin et al. 1994) we did not find any null point in the NLFFF extrapolation around the location of the flaring structure (see also the bottom panel of Fig. 16 where the colour-coded field lines show large field values everywhere). In order to clarify this point, we study the quasiseparatrix layers (QSLs) that can be obtained from the NLFFF extrapolation. The left panel of Fig. 17 shows the distribution of the squashing degree $Q$ at the photospheric level, while in the right panel we trace the separatrix field lines at the location of the bald patches (BP, i.e. locations where the magnetic field is tangent to the photosphere and concave-up, Titov et al. 1993). The resulting field structure (right panel of Fig. 17) includes both a linear structure along the PIL and a dome structure.

The BP field lines thus show that the linear and dome features are pseudo-spine and pseudo-fan structures, but are not a true spine/fan topology coming from a null. However, since they are separatrices, as discussed in (Démoulin 2006), they are preferential sites for the build-up of thin current sheets and hence for magnetic reconnection. This has been previously shown in, for example, Billinghurst et al. (1993), Pariat et al. (2009) and references therein. In the NLFFF extrapolation, such currents also include the volume around the pseudo-spine structure, as shown in the bottom panel of Fig. 18. The shape of the field lines associated with the brightening is identified with a pseudo-fan/spine albeit in the absence of a coronal null point, as shown in Fig. 16. From the QSL spatial distribution in Fig. 17, it is therefore evident that the semi-circular structure appearing as a semi-circular ribbon in the observations is actually the photospheric anchoring of the dome magnetic structure. Moreover, the location of the kernels $\mathrm{K} 1$ and $\mathrm{K} 2$ shown in Sect. 2.2 can be equally associated with the dome of the pseudo-fan structure, cf. Figs. 6 and 17.

Hence, on the grounds of the NLFFF extrapolation and the QSL study obtained from it, we argue that the homologous flares 

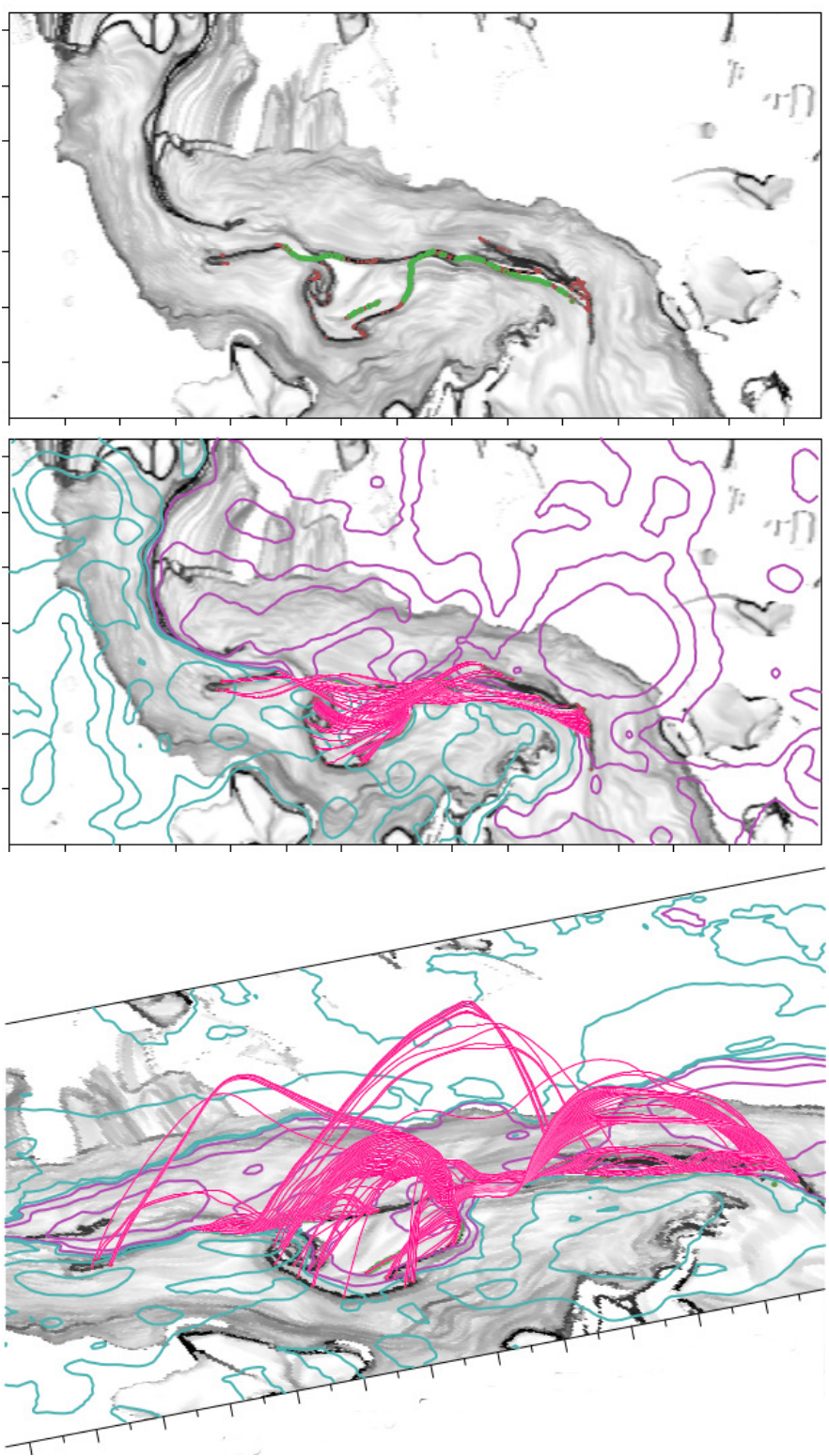

Fig. 17. Top panel: photospheric distribution of squashing factor $Q$, with the bald patch locations (green dots) overlaid. Middle panel: photospheric squashing degree, with bald-patch separatrix field lines and isocontours of $B_{z}$ overlaid. The bald-patch separatrix field lines are traced from the bald patch locations (green dots) shown on the top panel. Bottom panel: side view of the bald-patch separatrix lines.

observed as the repeated brightenings of the $\mathrm{K} 1$ and $\mathrm{K} 2$ kernels are the result of bald patch reconnection involving one single magnetic structure, which is likely to be excited by the same recurrent mechanism (e.g. flux emergence and/or shearing motion in the pseudo-fan area). The role of BPs in active events such as flares, surges, jets and brightenings, has been extensively studied, see e.g. Aulanier et al. (1998), Mandrini et al. (2002), Peter et al. (2014). Reconnection at BPs has been observed, in the framework of flux emergence, in several simulations: Archontis \& Hood (2009), Cheung et al. (2010), Archontis et al. (2013), Takasao et al. (2015). The shape of the brightenings is reminiscent of a typical null/fan topology but no null is present in the coronal field: in the relevant volume connecting the fanlike and the spine-like structure the magnetic field is of the order of $\sim 100 \mathrm{G}$ (see Fig. 16). This is also confirmed by the absence
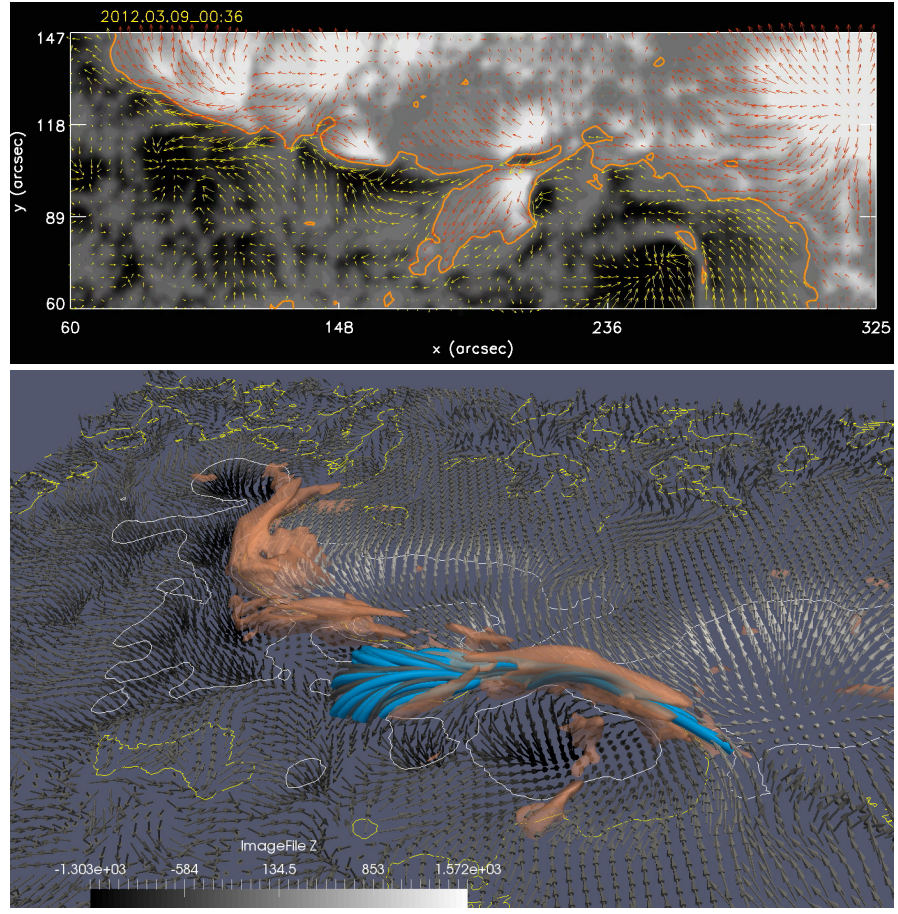

Fig. 18. Top panel: photospheric field just before the beginning of the C1.0 class flare as observed by SDO/HMI. The red/yellow arrows represent the transverse components of the magnetic field starting from negative/positive $B_{z}$ values. The orange line is the PIL. Bottom panel: spine-like structure and its surrounding current envelope. The current density is represented by the brownish semi-transparent isosurface (at $26 \%$ of its peak volume).

in the vector magnetogram of the parasitic polarity, which would correspond to the anchoring of the inner spine in a proper nullpoint topology, as the top panel of Fig. 18 shows. Such a configuration is theoretically possible if a null point is located below the photospheric plane.

\section{Discussion}

In this work, we have presented an analysis of a series of recurrent solar flares based on spectroscopic, multiwavelength imaging, and photospheric magnetic field observations. These observations provide a powerful tool to compare the physical parameters of the plasma in different flares occurring in the same $\mathrm{AR}$ and to investigate the mechanisms behind their homologous nature.

We analysed three confined C-class flares (C1.0, C2.0 and C4.7), which were observed during a single EIS observing sequence, and compared the results with the observations of the last M1.8 class eruptive flare seen during a full-spectrum EIS study, as reported by Doschek et al. (2013).

All the recurrent flares occurred within a short period of time $(\approx 3 \mathrm{~h}$ and a half in total) and show a very similar plasma morphology and magnetic field configuration. In particular, they are all characterized by the appearance of bright and compact footpoint sources of upflowing plasma along the quasicircular ribbon and the continuous activation of a spine-like hot plasma structure strongly emitting in Fe XXI and Fe XXIII emission $(\approx 10 \mathrm{MK})$, as observed by AIA and EIS.

By comparing the upflows measured at the flare footpoints in the EIS Fe XXIII line, we found similar values of blueshifts 
velocity (of the order of $150-200 \mathrm{~km} \mathrm{~s}^{-1}$ ) in the $\mathrm{C} 2.0, \mathrm{C} 4.7$ (at the footpoint K1) and M1.8 class flares. The Fe XXIII emission is too faint to be observed at the beginning of the smallest C1.0 flare impulsive phase and is only observed around the peak phase, when the evaporation phase is likely to have already been concluded.

However, the cadence of the EIS raster (around $3 \mathrm{~min}$ ) is comparable to the duration of the impulsive phase of the three confined flares under study, which is around 3-4 min, as shown by the GOES light curves in Fig. 1. Hence, it would not be surprising if higher upflow velocities were reached during the chromospheric evaporation phase but were missed by the EIS slit. We emphasize that the spectrometer rasters over the flare footpoints at different times into the impulsive phase of each flare. For instance, high velocities $\left(\approx 200 \mathrm{~km} \mathrm{~s}^{-1}\right)$ are measured during the $\mathrm{C} 2.0$ class flare at footpoint $\mathrm{K} 1$ at the very beginning of the impulsive phase of this flare (around $177 \mathrm{~s}$ after the beginning of the flare as measured in the soft X-ray flux). During the C4.7 class flare, blueshifted velocities of around $145 \mathrm{~km} \mathrm{~s}^{-1}$ are measured by EIS at the same K1 footpoint around $300 \mathrm{~s}$ after the beginning of the flare as measured in the soft X-ray flux. In addition, Doschek et al. (2013) measured 150-200 $\mathrm{km} \mathrm{s}^{-1}$ upflows during the M-class flare over almost the entire raster between 03:24:52 UT to 03:40:42 UT. This seems to suggest that the magnitude of the maximum upflows are indeed similar (within the range 150-200 $\mathrm{km} \mathrm{s}^{-1}$ ) for the C2.0, C4.7 (at footpoint K1) and M1.8 recurrent flares studied here. Simultaneously, the blueshifts of the Fe XVI line at $3 \mathrm{MK}$ are observed to be within the range of $\approx 40-60 \mathrm{~km} \mathrm{~s}^{-1}$ at the $\mathrm{K} 1$ footpoint of the C2.0 class flare and at the ribbon of the M-class flare. The C4.7 class flare shows a large Fe XVI blueshift velocity of around $76 \mathrm{~km} \mathrm{~s}^{-1}$ at the footpoint $\mathrm{K} 1$; however, this value has a large uncertainty due to the double Gaussian fit of the line profile.

Moreover, the observed Fe XXIII and Fe XVI blueshifts during the $\mathrm{C} 2.0$ confined flare, where, in contrast to the M-class flare, two footpoint sources K1 and K2 could be clearly distinguished, seem to be larger in K1 than in K2. The difference in the evaporating flows from the two footpoints is lower for the C4.7 class flare. Different evaporating flows at the two kernels could be related to an asymmetry in the mechanism that is responsible for the heating of the plasma at the chromospheric loop footpoints; that is, for instance, differently accelerated electron beams in the context of the collisional thick-target flare model. Asymmetric HXR footpoint sources have often been observed (e.g. Li et al. 1997; Aschwanden et al. 1999) and are generally interpreted as due to a difference in strength in the photospheric magnetic field at the two footpoints. In the case of a weaker magnetic field, the electrons are in fact less affected by magnetic mirror and are more able to reach and heat the chromosphere. However, some flares have been observed not to fit in this scenario (e.g. Asai et al. 2002). In addition, asymmetric HXR sources (and thus different evaporation) at the footpoints could also be explained by the injection of an electron beam along one of the flare loops rather than at the loop apex (Falewicz \& Siarkowski 2007).

Based on the use of the Fe XIV 264/274 $\AA$ density-sensitive line ratio, the EIS spectroscopic observations also provide estimates for the electron number density of the $2 \mathrm{MK}$ plasma during the recurrent flares. Our measurements suggest that, for all the flares under study, the electron density increases by around an order of magnitude or more at the flare footpoints compared to the background regions during the impulsive phase, in agreement with previous studies (e.g. Del Zanna et al. 2011a; Polito et al. 2016). For each flare, the densities at the two footpoints are similar within the errors and change over time, as shown in the density maps in Figs. 12, A.2 and A.1. In addition, within the errors, lower values of density are observed during the C1.0 flare $\left(\approx 10^{10.0-10.2} \mathrm{~cm}^{-3}\right)$ than the C2.0 $\left(\approx 10^{10.4-10.7} \mathrm{~cm}^{-3}\right)$ and the C4.7 $\left(10^{11} \mathrm{~cm}^{-3}\right.$ or more $)$ class flares at both footpoints. Taking the relative uncertainty in the measurements into account, the maximum value of density reached during the $\mathrm{C} 4.7$ class flare is higher than that of the $\mathrm{C} 2.0$ flare at the footpoint $\mathrm{K} 1$, while it is similar within the errors at the footpoint K2. On the other hand, measurements of the density over time show that very high values (above the density sensitivity limit) are obtained for a longer period time during the impulsive phase of the C4.7 flare (see Fig. 12). In addition, the M-class flare studied by Doschek et al. (2013) also shows densities reaching the $10^{11} \mathrm{~cm}^{-3}$ high density limit. It should be noted that a correlation between emission measure $(\mathrm{EM})$ of the plasma $\left(\mathrm{EM} \propto N_{\mathrm{e}}^{2}\right)$ and the soft X-ray flux was also observed by previous authors; see e.g. Feldman et al. (1996), Battaglia et al. (2005).

Given that similar velocities are observed during flares of different size, an increase in the electron number density with larger peak soft X-ray flux would indeed be expected to satisfy conservation of momentum of the plasma evaporating from the flare kernels and filling the flare loops (in the context of the standard model of flares). For instance, assuming the same size for the C2.0 and C4.7 flare footpoint sources (as observed within the EIS spatial resolution), the ratio between the peak soft X-ray energy of the two flares is around a factor of $\approx 2.3$ (4.7/2.0) from the GOES measurements. This value is consistent with the ratio of density values measured during the two flares (i.e. $10^{11} / 10^{10.6} \approx 2.5$ ).

Finally, the temperature diagnostics based on the AIA $131 / 94 \AA$ line ratio show that high temperature plasma $(\approx 10 \mathrm{MK})$ is observed at the flare footpoints and spine-like structure, as also confirmed by the spectroscopic measurements in the Fe XXIII line with EIS. In addition, by comparing the temperature diagnostics during the three confined flares, we observe that slightly higher temperatures (around $10 \%$ higher with a relative uncertainty of $1-2 \%$ ) are reached a few minutes before the peak of the C4.7 flare than those of the other two flares. It is emphasized that an increase in the electron temperature with peak soft $\mathrm{X}$-ray flux of the flare is in agreement with previous results by, for example, Feldman et al. (1996). However, since the AIA images are badly saturated during the peak of the C2.0 and C4.7 class flares, reliable temperature measurements and conclusive comparisons cannot be obtained.

We performed a NLFFF extrapolation of the 3D magnetic field configuration in the corona to understand the context of the observed events and investigate the mechanism responsible for the homologous flares. The NLFFF extrapolation catches the global topology and provides a good agreement with the location of the quasi-circular ribbon and the $10 \mathrm{MK}$ spine-like structure observed in AIA $131 \AA$. The extrapolation provides the magnetic field context that is sufficient to interpret and explain the locations of all brightenings involved in the (non-eruptive) flares (kernels, spine and semi-circular brightenings) that have been studied.

The semi-circular ribbon shape is similar to those created by the presence of a coronal null point, plus an associated spine-like structure connected to the circular structure. However, there is no coronal null point, not just because it is absent in the NLFFF extrapolation, but because the observed photospheric field does not show the parasitic polarity necessary for its existence in the corona. Instead, bald patch reconnection activates a magnetic 
structure consisting of a pseudo-fan and pseudo-spine. Such a configuration is conceivable as the geometrical prolongation in the corona of a sub-photospheric null point structure. On the basis of the NLFFF and the QSL study, we argue that the homologous flares were all generated by a repetition of the same process, namely bald patch reconnection.

We interpret the time evolution of the GOES fluxes in Fig. 1 and the temperature distribution estimations in Fig. 14 as indications of a progressive increase in energy of the events considered. Within the validity of the linear force-free theory, Valori et al. (2015) derives the relation

$$
\Delta \mathcal{E}_{\text {free }} \simeq \frac{1}{4 \pi} H_{\mathrm{m}} \Delta \alpha
$$

which relates the drop in free energy, $\Delta \mathcal{E}_{\text {free }}$, due to a flare to the change in the force-free parameter $\Delta \alpha$ and the relative magnetic helicity of the whole active region, $H_{\mathrm{m}}$. The considered homologous flares are repetitions of roughly the same reconnection process, hence, in the spirit of the linear theory, we can represent the change in connectivity producing them as a similar change $\Delta \alpha$ in the linear parameter characterizing the field. Since the active region is in an emerging phase with increasing energy (and helicity) over several days (Dhakal \& Zhang 2016), then Eq. (1) shows that the same (small) drop in $\Delta \alpha$ generates flares that are higher and higher in energy, because of the increased accumulated helicity in time. In this sense, Eq. (1) provides a simple explanation why homologous flares often show a progressive increase in energy, as it does for the case under examination; see Fig. 1. However, this simple explanation is based on linear theory, and inherits its limited range of applicability, especially when dealing with fully non-linear configurations, as in the case treated here.

The EIS observations in this work, coupled with the modelling of the magnetic field structure based on the NLFFF extrapolation, provide important information about the physical condition of the plasma and the reconnection process during the recurrent flares. In particular, the plasma parameters obtained by the analysis of the EIS spectra provide constraints for hydrodynamical models using different values of total energy inputs (for each flare) and based on different heating mechanisms, such as electron beams or conduction fronts, assuming different time profiles and geometries for the heating injection.

In order to confirm the results of the comparative analysis presented in this work, future spectroscopic studies of recurrent flares would need higher temporal cadence observations at the same footpoint source. This could best be achieved with an EIS sit-and-stare rather than a raster observing mode. On the other hand, raster observations offer the advantage of observing both footpoint sources, but a higher cadence than that used in this study would still be desirable.

Acknowledgements. V.P. acknowledges support from the Isaac Newton Studentship and the Cambridge Trust. GDZ and HEM acknowledge support from the STFC and the RS Newton Alumni Programme. J.D. acknowledges support from the Grant Agency of the Czech Republic (GA CR), projects No. P209/12/1652, and 16-17586S. V.P., G.D.Z., H.E.M., and J.D. acknowledge support from the Royal Society via the Newton Alumni programme. G.V. acknowledges the support of the Leverhulme Trust Research Project Grant 2014-051. Hinode is a Japanese mission developed and launched by ISAS/JAXA, with NAOJ as domestic partner and NASA and STFC (UK) as international partners. It is operated by these agencies in co-operation with ESA and NSC (Norway). AIA and HMI data are courtesy of NASA/SDO and their respective science teams. CHIANTI is a collaborative project involving researchers at the universities of Cambridge (UK), George Mason and Michigan (USA).

\section{References}

Antonucci, E., Gabriel, A. H., Acton, L. W., et al. 1982, Sol. Phys., 78, 107 Archontis, V., \& Hood, A. W. 2009, A\&A, 508, 1469

Archontis, V., Hood, A. W., \& Tsinganos, K. 2013, ApJ, 778, 42

Asai, A., Masuda, S., Yokoyama, T., et al. 2002, ApJ, 578, L91

Aschwanden, M. J., Fletcher, L., Sakao, T., Kosugi, T., \& Hudson, H. 1999, ApJ, 517,977

Aulanier, G., Démoulin, P., Schmieder, B., Fang, C., \& Tang, Y. H. 1998, Sol. Phys., 183, 369

Aulanier, G., Janvier, M., \& Schmieder, B. 2012, A\&A, 543, A110

Battaglia, M., Grigis, P. C., \& Benz, A. O. 2005, A\&A, 439, 737

Battaglia, M., Kleint, L., Krucker, S., \& Graham, D. 2015, ApJ, 813, 113

Billinghurst, M. N., Craig, I. J. D., \& Sneyd, A. D. 1993, A\&A, 279, 589

Boerner, P., Edwards, C., Lemen, J., et al. 2012, Sol. Phys., 275, 41

Boerner, P. F., Testa, P., Warren, H., Weber, M. A., \& Schrijver, C. J. 2014, Sol. Phys., 289, 2377

Brosius, J. W. 2003, ApJ, 586, 1417

Brosius, J. W. 2013, ApJ, 762, 133

Carmichael, H. 1964, NASA SP, 50, 451

Cheung, M. C. M., Rempel, M., Title, A. M., \& Schüssler, M. 2010, ApJ, 720, 233

Chintzoglou, G., Patsourakos, S., \& Vourlidas, A. 2015, ApJ, 809, 34

Culhane, J. L., Harra, L. K., James, A. M., et al. 2007, Sol. Phys., 243, 19

De Pontieu, B., Title, A. M., Lemen, J. R., et al. 2014, Sol. Phys., 289, 2733

Del Zanna, G., \& Woods, T. N. 2013, A\&A, 555, A59

Del Zanna, G., Berlicki, A., Schmieder, B., \& Mason, H. E. 2006, Sol. Phys., 234, 95

Del Zanna, G., Mitra-Kraev, U., Bradshaw, S. J., Mason, H. E., \& Asai, A. 2011a, A\&A, 526, A1

Del Zanna, G., O’Dwyer, B., \& Mason, H. E. 2011b, A\&A, 535, A46

Démoulin, P. 2006, Adv. Space Res., 37, 1269

Démoulin, P., Hénoux, J. C., \& Mandrini, C. H. 1994, A\&A, 285, 1023

Demoulin, P., Henoux, J. C., Priest, E. R., \& Mandrini, C. H. 1996, A\&A, 308, 643

Dhakal, S. K., \& Zhang, J. 2016, in AAS/Solar Physics Division Meeting, 47, 11.02

Doschek, G. A., Kreplin, R. W., \& Feldman, U. 1979, ApJ, 233, L157

Doschek, G. A., Warren, H. P., \& Young, P. R. 2013, ApJ, 767, 55

Doschek, G. A., Warren, H. P., Dennis, B. R., Reep, J. W., \& Caspi, A. 2015, ApJ, 813, 32

Dudík, J., Janvier, M., Aulanier, G., et al. 2014, ApJ, 784, 144

Dudík, J., Polito, V., Janvier, M., et al. 2016, ApJ, 823, 41

Emslie, G. A., \& Alexander, D. 1987, Sol. Phys., 110, 295

Falewicz, R., \& Siarkowski, M. 2007, A\&A, 461, 285

Feldman, U., Doschek, G. A., Behring, W. E., \& Phillips, K. J. H. 1996, ApJ, 460, 1034

Fuhrmann, M., Seehafer, N., \& Valori, G. 2007, A\&A, 476, 349

Hannah, I. G., Hudson, H. S., Battaglia, M., et al. 2011, Space Sci. Rev., 159, 263

Hao, Q., Guo, Y., Dai, Y., et al. 2012, A\&A, 544, L17

Harrison, R. A., Sawyer, E. C., Carter, M. K., et al. 1995, Sol. Phys., 162, 233

Hirayama, T. 1974, Sol. Phys., 34, 323

Hoeksema, J. T., Liu, Y., Hayashi, K., et al. 2014, Sol. Phys., 289, 3483

Janvier, M., Aulanier, G., Pariat, E., \& Démoulin, P. 2013, A\&A, 555, A77

Janvier, M., Aulanier, G., \& Démoulin, P. 2015, Sol. Phys., 290, 3425

Kahler, S. W. 1982, J. Geophys. Res., 87, 3439

Kamio, S., Hara, H., Watanabe, T., Fredvik, T., \& Hansteen, V. H. 2010, Sol. Phys., 266, 209

Kopp, R. A., \& Pneuman, G. W. 1976, Sol. Phys., 50, 85

Kouloumvakos, A., Patsourakos, S., Nindos, A., et al. 2016, ApJ, 821, 31

Li, J., Metcalf, T. R., Canfield, R. C., Wülser, J.-P., \& Kosugi, T. 1997, ApJ, 482, 490

Mandrini, C. H., Démoulin, P., Schmieder, B., Deng, Y. Y., \& Rudawy, P. 2002, A\&A, 391, 317

Masson, S., Pariat, E., Aulanier, G., \& Schrijver, C. J. 2009, ApJ, 700, 559

Metcalf, T. R., Derosa, M. L., Schrijver, C. J., et al. 2008, Sol. Phys., 247, 269

Milligan, R. O. 2015, Sol. Phys., 290, 3399

Milligan, R. O., Gallagher, P. T., Mathioudakis, M., et al. 2006, ApJ, 638, L117

Nitta, N. V., \& Hudson, H. S. 2001, Geophys. Res. Lett., 28, 3801

O’Dwyer, B., Del Zanna, G., Mason, H. E., Weber, M. A., \& Tripathi, D. 2010, A\&A, 521, A21

Pariat, E., \& Démoulin, P. 2012, A\&A, 541, A78

Pariat, E., Masson, S., \& Aulanier, G. 2009, ApJ, 701, 1911

Patsourakos, S., Georgoulis, M. K., Vourlidas, A., et al. 2016, ApJ, 817, 14

Peter, H., Tian, H., Curdt, W., et al. 2014, Science, 346, 1255726 
A\&A 601, A39 (2017)

Petkaki, P., Del Zanna, G., Mason, H. E., \& Bradshaw, S. J. 2012, A\&A, 547, A25

Polito, V., Reep, J. W., Reeves, K. K., et al. 2016, ApJ, 816, 89

Priest, E. R., \& Démoulin, P. 1995, J. Geophys. Res., 100, 23443

Reeves, K. K., Warren, H. P., \& Forbes, T. G. 2007, ApJ, 668, 1210

Reid, H. A. S., Vilmer, N., Aulanier, G., \& Pariat, E. 2012, A\&A, 547, A52

Romano, P., Zuccarello, F., Guglielmino, S. L., et al. 2015, A\&A, 582, A55

Scherrer, P. H., Schou, J., Bush, R. I., et al. 2012, Sol. Phys., 275, 207

Schrijver, C. J., DeRosa, M. L., Metcalf, T., et al. 2008, ApJ, 675, 1637

Simões, P. J. A., Fletcher, L., Hudson, H. S., \& Russell, A. J. B. 2013, ApJ, 777, 152

Sturrock, P. A. 1968, Astron. J. Suppl., 73, 78

Sun, X., Hoeksema, J. T., Liu, Y., et al. 2013, ApJ, 778, 139
Syntelis, P., Gontikakis, C., Patsourakos, S., \& Tsinganos, K. 2016, A\&A, 588, A16

Takasao, S., Fan, Y., Cheung, M. C. M., \& Shibata, K. 2015, ApJ, 813, 112

Teriaca, L., Falchi, A., Cauzzi, G., et al. 2003, ApJ, 588, 596

Titov, V. S., Priest, E. R., \& Demoulin, P. 1993, A\&A, 276, 564

Titov, V. S., Hornig, G., \& Démoulin, P. 2002, J. Geophys. Res. (Space Physics), 107,1164

Valori, G., Kliem, B., Török, T., \& Titov, V. S. 2010, A\&A, 519, A44

Valori, G., Démoulin, P., \& Pariat, E. 2012, Sol. Phys., 278, 347

Valori, G., Démoulin, P., Pariat, E., \& Masson, S. 2013, A\&A, 553, A38

Valori, G., Romano, P., Malanushenko, A., et al. 2015, Sol. Phys., 290, 491

Warren, H. P., \& Doschek, G. A. 2005, ApJ, 618, L157

Young, P. R., O’Dwyer, B., \& Mason, H. E. 2012, ApJ, 744, 14 
V. Polito et al.: Analysis and modelling of recurrent solar flares observed with Hinode/EIS on March 9, 2012

\section{Appendix A: Density diagnostics in the $\mathrm{C} 2.0$ and $\mathrm{C} 1.0$ class flares}

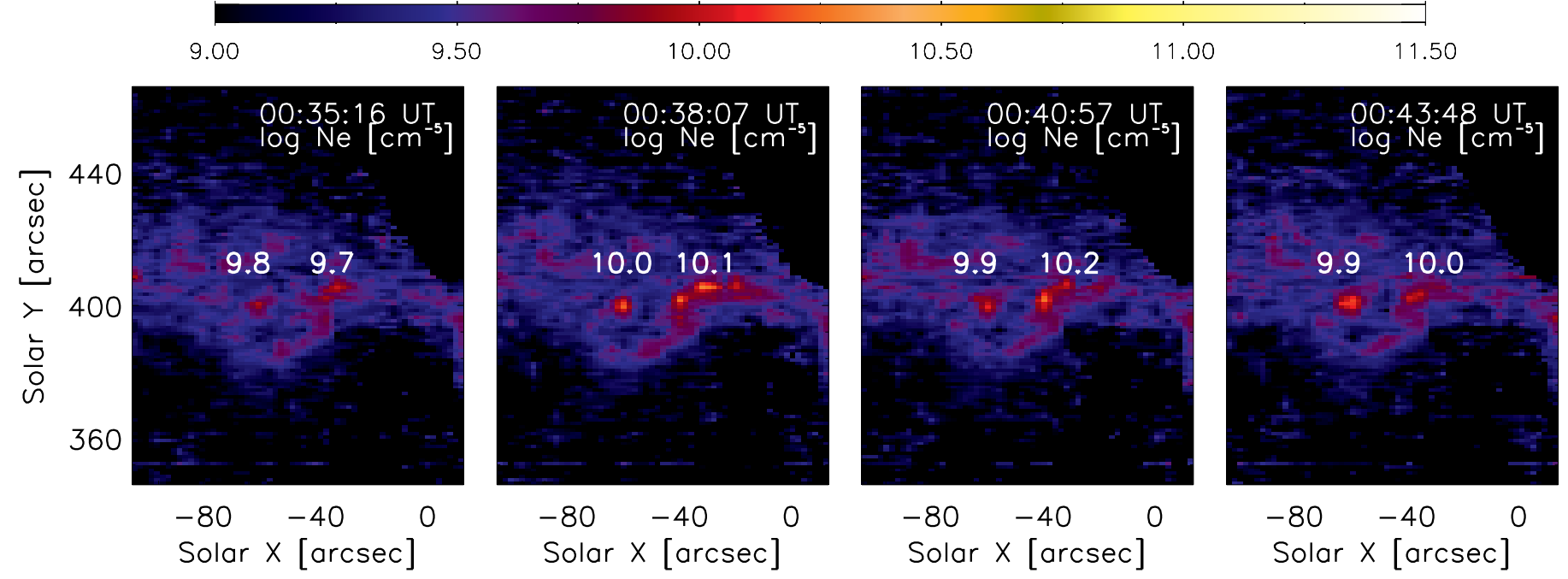

Fig. A.1. Density maps (in log scale) for different times during the C1.0 class flare. See caption of Fig. 12 for more details.

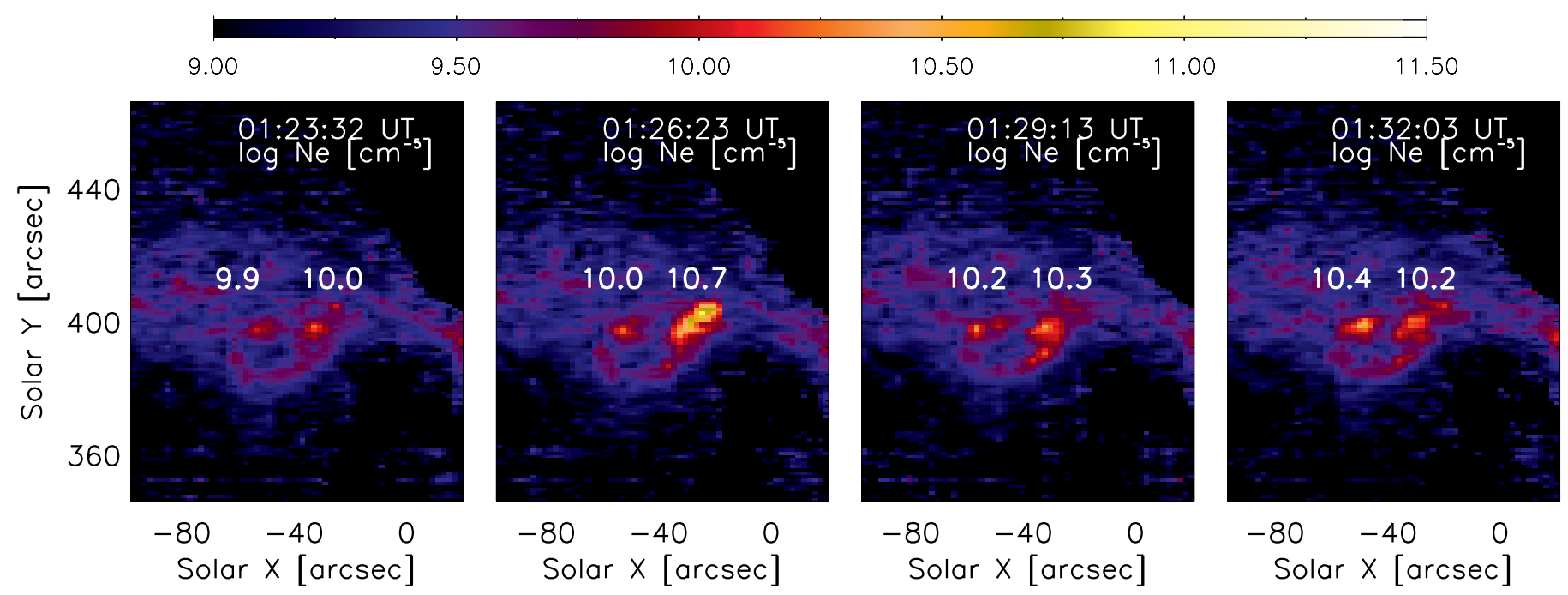

Fig. A.2. Density maps (in log scale) for different times during the C2.0 class flare. See caption of Fig. 12 for more details. 